\title{
Statistical theory of diffusion-reaction processes in the system "metal-adsorbate-gas"
}

\author{
P.P.Kostrobii ${ }^{1}$, B.M.Markovych ${ }^{1}$, Yu.K.Rudavskii ${ }^{1}$, \\ M.V.Tokarchuk ${ }^{2}$ \\ 1 State University "Lvivska Politehnika", \\ 12 Bandera Str., 79646 Lviv, Ukraine \\ 2 Institute for Condensed Matter Physics \\ of the National Academy of Sciences of Ukraine, \\ 1 Svientsitskii Str., 79011 Lviv, Ukraine
}

Received March 1, 2001

\begin{abstract}
We present a statistical theory for diffusion-reaction processes of gaseous mixture in the system "metal-adsorbate-gas". The theory is based on an equal consideration of electron-electron, electron-atom and atom-atom interactions between adsorbed, non-adsorbed atoms and atoms of metal surface. On a metal surface, the bimolecular reactions of the $A+B \leftrightarrow A B$ type are possible between the adsorbed atoms which is typical of catalytic processes. By means of Zubarev nonequilibrium statistical operator, the system of transport equations is obtained for a consistent description of electronic kinetic and diffusion-reaction atomic processes.
\end{abstract}

Key words: adsorbate, diffusion-reaction atomic processes, transport equations

PACS: $05.30 . \mathrm{Ch}, 05.20 . \mathrm{Dd}, 73.40 . \mathrm{Gk}, 68.45 . \mathrm{Kg}$

\section{Introduction}

Processes of adsorption, desorption, diffusion of atoms, ions, polar and magnetic molecules or clusters on the surfaces of metals, insulators, semiconductors play one of central roles in the development of nanostructural thin film technologies for microelectronics and optoelectronics. Diffusion processes, adsorption and desorption mechanisms are also decisive in catalytic reactions on active surfaces where the structure and the electronic structure play a central role. Electronic processes on a metal surface, which create local electric fields in catalytic reactions are also the promoters of dissociation-association processes of gas molecules. All these phenomena make the study of mechanics of different catalytic reactions much more complicat- 
ed. Besides, to understand them, a rigorous and detailed study of electronic kinetic and diffusion-reaction atom-molecular processes should be carried out. Such processes and phenomena are the subject of an intensive experimental and theoretical study in solid state physics. Nowadays, experimental methods of investigation such as scanning-tunnelling-microscopy (STM), scanning-tunnelling-spectroscopy (STS), field-ion-microscopy (FIM), and their modifications provide each time a more detailed information about the electronic structure, diffusion processes, structural transformations on the surfaces of metals, insulators, semiconductors, high temperature superconductors [1-7]. A more sequential theory of atom transport at scanning by tunnelling electrons with taking into account the mechanisms of atom heat oscillations and substrate phonon oscillations and with the use of transfer Hamiltonian "substrate-adsorbate-tip" was presented in papers [8-11]. Of course, the processes of atom and molecule transport on the surface of a solid state, no matter whether the STM investigations are pursued or not, critically depend both on the nature of interactions between them, which can be dipolar or magnetic, and on the state of the substrate: paramagnetic, ferromagnetic, ferrimagnetic, etc. Furthermore, for such spatially inhomogeneous systems one has another topical problem: the description of quantum transport processes at small times with taking into account the initial states and non-Markovian memory effects; the description of chemical catalytic reactions on a metal surface. One approach for the construction of quantum kinetic equations with taking into account the initial states and non-Markovian memory effects was recently suggested based on the mixed Green functions [12,13]. Processes of atom and molecule transport on the surface of a solid can be described here based on the theory of surface diffusion $[14,15]$, or on kinetic equations $[16,17]$.

In the present paper, we present generalized transport equations of a consistent description of electron kinetic and atomic diffusion-reaction processes in a system "metal-adsorbate-gas". To this end, we use the nonequilibrium statistical operator (NSO) method by D.N.Zubarev $[12,18]$ and obtain a kinetic equation for one-electron density matrix and relevant to this relation diffusion-reaction equations for adsorbed and non-adsorbed gas atoms on a metal surface.

\section{Nonequilibrium statistical operator and transport equations of electrons and atoms of a system "metal-adsorbate-gas"}

For a consistent description of electron kinetics of processes on a metal surface with adsorbed gas atoms or molecules one needs to take into account many peculiarities connected with screening effects and surface diffusion. We consider here the system "metal-adsorbate-gas". Gas molecules become polarized and can dissociate near the metal surface in nonhomogeneous electric field, which is produced by conduction electrons and localized electrons (for example $d$-electrons of transition metals) as well as by metal surface ions. Finally, due to the interaction, the dissociation products are adsorbed on the metal surface. This is the dissociation mechanism of gas molecules in numerous catalytic reactions (especially, ammoniac catalysis). Then, the dissociation products of different molecules, which are adsorbed on a met- 
al surface, join the chemical reactions with energy threshold, which is sufficiently lower than for the reactions in a volume state without catalyser. Henceforth, it is more energetically preferable for the reaction products to leave the surface. Modern catalytic reactions of the surface are mainly bimolecular

$$
A+B \leftrightarrow A B
$$

though metal surface atoms actively participate therein. This is displayed through the electron-ion-molecular interactions.

Let us suppose that after the interaction of gas atoms or molecules with the surface, some portion thereof is adsorbed. Let $N_{a}$ be the total number of non-adsorbed atoms, whereas $N_{\bar{a}}$ be the number of atoms adsorbed on a metal surface, $N_{e}$ be the total number of electrons, and $N_{s}$ be the number of ions of a metal. The total Hamiltonian of such a system reads:

$$
H=H_{e}+H_{i}^{\mathrm{int}}+H_{a}+H_{a}^{\mathrm{int}}+\sum_{\substack{\alpha=a, s, \bar{a} \\ 1 \leqslant f \leqslant N_{\alpha}}} U_{\alpha}\left(z_{f}\right)+H_{\mathrm{reac}}
$$

where

$$
H_{a}=\sum_{j=1}^{N_{a}} \frac{p_{j}^{2}}{2 m_{a}}+\frac{1}{2} \sum_{j, j^{\prime}}^{N_{a}} V_{a a}\left(\left|\mathbf{r}_{j}-\mathbf{r}_{j^{\prime}}\right|\right)
$$

is the Hamiltonian of a gas subsystem, where $\mathbf{p}_{j}$ - is a gas atom momentum, $m_{a}$ - is its mass, $V_{a a}\left(\left|\mathbf{r}_{j}-\mathbf{r}_{j^{\prime}}\right|\right)$ - is a binary interaction potential of gas atoms on a distance $\left|\mathbf{r}_{j}-\mathbf{r}_{j^{\prime}}\right|$. Gas atoms interact with electrons of a subsystem "metal - adsorbate", metal surface ions and adsorbed atoms. Let us denote this part of interaction energy $H_{a}^{\text {int }}$ :

$$
H_{a}^{\mathrm{int}}=\sum_{j, l}^{N_{a}, N_{e}} V_{a e}\left(\left|\mathbf{r}_{j}-\mathbf{r}_{l}\right|\right)+\sum_{j, f}^{N_{a}, N_{s}} V_{a s}\left(\left|\mathbf{r}_{j}-\mathbf{R}_{f}\right|\right)+\sum_{j, j^{\prime}}^{N_{a}, N_{\bar{a}}} V_{a \bar{a}}\left(\left|\mathbf{r}_{j}-\mathbf{R}_{j^{\prime}}\right|\right),
$$

where $V_{a e}$ is an electron-atom potential of interaction, $V_{a s}$ is a potential of interaction of a gas atom with an ion of a metal surface, $V_{a \bar{a}}$ is a potential of interaction of a gas atom with an adsorbed atom. Electrons in a subsystem "metal - adsorbate - gas" interact between themselves, between metal ions with microscopic charge density $\hat{\rho}_{s}(\mathbf{R})$, between the adsorbed on a metal surface gas atoms with microscopic charge density $\hat{\rho}_{\bar{a}}(\mathbf{R})$. The Hamiltonian of an electron subsystem $H_{e}$ then reads:

$$
H_{e}=-\frac{\hbar^{2}}{2 m_{e}} \sum_{l=1}^{N_{e}} \Delta_{l}+\frac{1}{2} \sum_{l \neq l^{\prime}} \frac{e^{2}}{\left|\mathbf{r}_{l}-\mathbf{r}_{l^{\prime}}\right|}-\sum_{l=1}^{N_{e}} \int \mathrm{d} \mathbf{R} \frac{e \hat{\rho}_{s}(\mathbf{R})}{\left|\mathbf{r}_{l}-\mathbf{R}\right|}-\sum_{l=1}^{N_{e}} \int \mathrm{d} \mathbf{R} \frac{e \hat{\rho}_{\bar{a}}(\mathbf{R})}{\left|\mathbf{r}_{l}-\mathbf{R}\right|}
$$

It consists of a kinetic energy, Coulomb interelectron interaction energy and the potentials of interactions of electrons with metal ions and the adsorbed atoms. $\mathbf{r}_{l}$ are electron coordinates, $\mathbf{R}_{f}$ are coordinates of corresponding ions and atoms. Aside 
from the electron subsystem, of great importance is to take into account interactions in an ion subsystem as well. Its Hamiltonian reads:

$$
\begin{aligned}
H_{\mathrm{i}}^{\mathrm{int}}= & -\frac{\hbar^{2}}{2 m_{a}} \sum_{f=1}^{N_{\bar{a}}} \Delta_{f}+\frac{1}{2} \int \mathrm{d} \mathbf{R} \mathrm{d} \mathbf{R}^{\prime} \frac{\hat{\rho}_{\bar{a}}(\mathbf{R}) \hat{\rho}_{\bar{a}}\left(\mathbf{R}^{\prime}\right)}{\left|\mathbf{R}-\mathbf{R}^{\prime}\right|} \\
& +\int \mathrm{d} \mathbf{R} \mathrm{d} \mathbf{R}^{\prime} \frac{\hat{\rho}_{\bar{a}}(\mathbf{R}) \hat{\rho}_{s}\left(\mathbf{R}^{\prime}\right)}{\left|\mathbf{R}-\mathbf{R}^{\prime}\right|}+\frac{1}{2} \int \mathrm{d} \mathbf{R} \mathrm{d} \mathbf{R}^{\prime} \frac{\hat{\rho}_{s}(\mathbf{R}) \hat{\rho}_{s}\left(\mathbf{R}^{\prime}\right)}{\left|\mathbf{R}-\mathbf{R}^{\prime}\right|}
\end{aligned}
$$

where kinetic energy of adsorbed gas atoms on a metal surface is also considered, other terms describe the interaction between metal ions, adsorbed atoms. $U_{\alpha}\left(z_{f}\right)$ is an inhomogeneous effective potential of a surface which is assembled by collective effects in a semilimited space, in our case in metal. $H_{\text {reac }}$ is the interaction Hamiltonian for the chemical reaction between adsorbed atoms or molecules on a metal surface:

$$
H_{\text {reac }}=\sum_{\bar{a}, \bar{b}, \bar{a}^{\prime}, \bar{b}^{\prime}}\left(\left\langle\bar{a}^{\prime}, \bar{b}^{\prime}\left|\Phi_{\text {reac }}\right| \bar{a}, \bar{b}\right\rangle \hat{q}_{\bar{a}^{\prime}}^{+} \hat{q}_{\bar{b}^{\prime}}^{+} \hat{q}_{\bar{a}} \hat{q}_{\bar{b}}+\left\langle\bar{a}^{\prime}, \bar{b}^{\prime}\left|\Phi_{\text {reac }}\right| \bar{a}, \bar{b}\right\rangle^{*} \hat{q}_{\bar{a}}^{+} \hat{q}_{\bar{b}}^{+} \hat{q}_{\bar{a}^{\prime}} \hat{q}_{\bar{b}^{\prime}}\right)
$$

with the amplitude $\left\langle\bar{a}^{\prime}, \bar{b}^{\prime}\left|\Phi_{\text {reac }}\right| \bar{a}, \bar{b}\right\rangle=\left\langle\bar{a}, \bar{b}\left|\Phi_{\text {reac }}\right| \bar{a}^{\prime}, \bar{b}^{\prime}\right\rangle$ of reaction between reagents $A, B$ and the reaction products $A B$ (we use the indices $\bar{a}, \bar{b}$ and $\bar{a}^{\prime} \bar{b}^{\prime}$ for the states of reagents $A, B$ (atoms or molecules) and for the states of atoms in the reaction product $A B)$. Here $\hat{q}_{\bar{a}^{\prime}}^{+}, \hat{q}_{\bar{b}^{\prime}}^{+}, \hat{q}_{\bar{a}}^{+}, \hat{q}_{\bar{b}}^{+}$and $\hat{q}_{\bar{a}^{\prime}}, \hat{q}_{\bar{b}^{\prime}}, \hat{q}_{\bar{a}}, \hat{q}_{\bar{b}}$ are the atom creation and annihilation operators for the states $\bar{a}^{\prime}, \bar{b}^{\prime}, \bar{a}$ and $\bar{b}$ of molecules $A B, A$ and $B$, correspondingly. We study the kinetics of an electron subsystem on a metal surface and diffusion-reaction processes of adsorbed and nonadsorbed gas atoms or molecules. In view of this, it is convenient to use the second quantization for electron subsystem in Hamiltonian (2.1) according to [19]. To this end, one needs to choose a proper basis of wave functions. Let us suppose that we know the solution to the Shrödinger equation for an electron

$$
\left[-\frac{\hbar^{2}}{2 m_{e}} \Delta+V_{e \alpha}\left(\mathbf{r}-\mathbf{X}_{\alpha}\right)\right] \psi_{\nu \alpha}\left(\mathbf{r}-\mathbf{X}_{\alpha}\right)=\varepsilon_{\nu \alpha} \psi_{\nu \alpha}\left(\mathbf{r}-\mathbf{X}_{\alpha}\right)
$$

in potential fields of a surface atom, non-adsorbed and adsorbed gas atoms. Here $\mathbf{X}_{\alpha}=\left(\mathbf{r}_{\alpha}, \mathbf{R}_{\alpha}\right)$ are Cartesian coordinates of particles. Eigen functions of equation (2.2) satisfy the conditions of orthogonality and completeness

$$
\begin{aligned}
\int \mathrm{d} \mathbf{R} \psi_{\nu}^{*}\left(\mathbf{r}-\mathbf{R}_{j}\right) \psi_{\mu}\left(\mathbf{r}^{\prime}-\mathbf{R}_{j}\right) & =\delta_{\nu \mu} \\
\sum_{\nu} \psi_{\nu}^{*}\left(\mathbf{r}-\mathbf{R}_{j}\right) \psi_{\nu}\left(\mathbf{r}^{\prime}-\mathbf{R}_{j}\right) & =\delta\left(\mathbf{r}-\mathbf{r}^{\prime}\right)
\end{aligned}
$$

for any $j=1, \ldots, N_{b},\{\nu, \mu, \xi\}$ are quantum numbers and $\varepsilon_{\nu \alpha}=\left(E_{\mu}, \varepsilon_{\nu}, E_{\xi}^{a d}\right)$ are eigen-values of electron energies. Let us use the set of functions $\psi_{\nu \alpha}\left(\mathbf{r}-\mathbf{X}_{\alpha}\right)=$ $\left(\psi_{v}\left(\mathbf{r}-\mathbf{R}_{f}\right), \varphi_{\mu}\left(\mathbf{r}-\mathbf{r}_{l}\right), \varphi_{\xi}^{a d}\left(\mathbf{r}-\mathbf{R}_{l}\right)\right)$ as a basis for the expansion of electron field 
operators:

$$
\begin{aligned}
\hat{\psi}(\mathbf{r}, \mathbf{s})= & \sum_{f=1}^{N_{+}} \sum_{\nu} \sum_{\sigma= \pm \hbar / 2} \psi_{\nu}\left(\mathbf{r}-\mathbf{R}_{f}\right) \chi_{\sigma}(s) \hat{a}_{f \nu \sigma}+\sum_{l=1}^{N_{a}} \sum_{\mu} \sum_{\sigma= \pm \hbar / 2} \varphi_{\mu}\left(\mathbf{r}-\mathbf{r}_{l}\right) \xi_{\sigma}(s) \hat{c}_{l \mu \sigma} \\
& +\sum_{l=1}^{N_{\bar{a}}} \sum_{\xi} \sum_{\sigma=\hbar / 2} \varphi_{\xi}^{a d}\left(\mathbf{r}-\mathbf{R}_{l}\right) \chi_{\sigma}(s) \hat{c}_{l \xi \sigma}^{a d}
\end{aligned}
$$

where $\chi_{\sigma}(s)$ are wave functions of an electron spin operator, $\sigma= \pm \hbar / 2$ are the electron spin projections on a quantization axis, $\mathbf{s}$ is a spin coordinate. $\hat{a}_{f \nu \sigma}, \hat{c}_{l \mu \sigma}$, $\hat{c}_{l \xi \sigma}^{a d}$ and $\hat{a}_{f \nu \sigma}^{+}, \hat{c}_{l \mu \sigma}^{+}, \hat{c}_{l \xi \sigma}^{(a d)+}$ are electron annihilation and creation operators, $\mathbf{R}_{f}$ denotes a position of surface atom, $\mathbf{r}_{l}$ - the same gas atom, $\mathbf{R}_{l}$ - for an adsorbed atom on a metal surface, respectively. Then, the Hamiltonian of electron subsystem taking into account (2.3) in the second quantization representation, reads:

$$
\begin{aligned}
H_{e}= & \sum_{\alpha, \nu, \sigma} \varepsilon_{\nu}^{\alpha} \hat{n}_{\nu, \sigma}^{\alpha}+\sum_{\alpha, \beta} \sum_{\sigma, \mu, \nu} t_{\mu \nu}^{\alpha \beta}\left(\hat{A}_{\nu \sigma}^{+\alpha} \hat{A}_{\mu \sigma}^{\beta}+\hat{A}_{\mu \sigma}^{+\beta} \hat{A}_{\nu \sigma}^{\alpha}\right) \\
& +\sum_{\substack{\alpha, \beta \\
\alpha^{\prime}, \beta^{\prime}}} \sum_{\nu \omega \sigma \mu \lambda \sigma^{\prime}} W_{\omega \lambda}^{\nu \mu}\left(\alpha, \beta ; \alpha^{\prime}, \beta^{\prime}\right) \hat{A}_{\nu \sigma}^{+\alpha} \hat{A}_{\mu \sigma^{\prime}}^{+\beta} \hat{A}_{\lambda \sigma^{\prime}}^{\alpha^{\prime}} \hat{A}_{\omega \sigma}^{\beta^{\prime}},
\end{aligned}
$$

where $\varepsilon_{\nu}^{\alpha}$ is one-electron energy in a field of a corresponding atom (surface, adsorbate, non-adsorbate). The operators $\hat{A}_{j \nu \sigma}^{+\alpha}$ assume the values $\hat{a}_{f \nu \sigma}^{+}, \hat{c}_{l \mu \sigma}^{+}, \hat{c}_{l \xi \sigma}^{(a d)+}$, whereas operators $\hat{A}_{j \mu \sigma}^{\alpha}$ are from the set $\hat{a}_{f \nu \sigma}, \hat{c}_{l \mu \sigma}, \hat{c}_{l \xi \sigma}^{a d}$ and are the electron creation and annihilation operators on the surface atom, gas atom, adsorbed atom on a metal surface, correspondingly.

$$
\hat{n}_{\nu \sigma}^{\alpha}=\sum_{j=1} \hat{A}_{j \nu \sigma}^{+\alpha} \hat{A}_{j \mu \sigma}^{\alpha}
$$

is the density operator of electrons in the field of corresponding atoms.

$$
\varepsilon_{v}^{\alpha}=\int \mathrm{d} \mathbf{r} \psi_{\nu}^{\alpha}(\mathbf{r})\left(-\frac{\hbar^{2}}{2 m_{e}} \Delta+U_{\alpha}(z)+V_{\alpha \alpha}(\mathbf{r})\right) \psi_{\nu}^{\alpha}(\mathbf{r}),
$$

where $V_{\alpha \alpha}(\mathbf{r})$ are corresponding potentials of electrons in the field of metal ions adsorbed, non-adsorbed gas atoms or molecules.

$$
t_{\nu \mu}^{\alpha \beta}=\int \psi_{\nu}^{\alpha}(\mathbf{r})\left(-\frac{\hbar^{2}}{2 m_{e}} \Delta+V_{\alpha \beta}(\mathbf{r})+U_{\alpha}(z)\right) \psi_{\mu}^{\beta}(\mathbf{r}) \mathrm{d} \mathbf{r}
$$

are matrix elements of the Hamiltonian. They describe processes of electron transitions in the field of corresponding atoms and ions.

$$
W_{\omega \lambda}^{\nu \mu}\left(\alpha \beta ; \alpha^{\prime} \beta^{\prime}\right)=\frac{1}{2} \iint \psi_{\nu}^{\alpha}(\mathbf{r}) \psi_{\omega}^{\beta^{\prime}}(\mathbf{r}) \frac{e^{2}}{\left|\mathbf{r}-\mathbf{r}^{\prime}\right|} \psi_{\mu}^{\beta}\left(\mathbf{r}^{\prime}\right) \psi_{\lambda}^{\alpha^{\prime}}\left(\mathbf{r}^{\prime}\right) \mathrm{d} \mathbf{r} \mathrm{d} \mathbf{r}^{\prime}
$$

is some Coulomb repulsive integral of the electrons, which are connected with corresponding atoms in accordance with (2.3). The analysis of the total Hamiltonian 
(2.4) of the electron subsystem can be made in detail in view of the hybridization processes between electron states of a surface and the atoms as well as the effects of interaction between electrons. Such an analysis should be made on the expansions in overlap integrals of orbitals of corresponding atoms similar to [20]. The current of the electrons between positions $\mathrm{l}$ and $\mathrm{j}$ in a system can be evaluated from the equation

$$
J_{l j}=\int \operatorname{Sp}\left(\hat{t}_{l j}\left(\hat{G}_{l j}^{+-}-\hat{G}_{j l}^{+-}\right)\right) \mathrm{d} E,
$$

where $\hat{G}_{l j}^{+-}, \hat{G}_{j l}^{+-}$are the spectral functions of time one-electron Green functions. These functions can be rewritten in the matrix form like this:

$$
\hat{G}_{l j}\left(1,1^{\prime}\right)=\left[\begin{array}{ll}
\hat{G}_{l j}^{++}\left(1,1^{\prime}\right) & \hat{G}_{l j}^{+-}\left(1,1^{\prime}\right) \\
\hat{G}_{l j}^{-+}\left(1,1^{\prime}\right) & \hat{G}_{l j}^{--}\left(1,1^{\prime}\right)
\end{array}\right]=\left[\begin{array}{ll}
\hat{g}_{l j}^{c}\left(1,1^{\prime}\right) & \hat{g}_{l j}^{<}\left(1,1^{\prime}\right) \\
\hat{g}_{l j}^{>}\left(1,1^{\prime}\right) & \hat{g}_{l j}^{a}\left(1,1^{\prime}\right)
\end{array}\right],
$$

where $\hat{g}_{l j}^{c}$ are causal, $\hat{g}_{l j}^{a}$ anticausal and $\hat{g}_{l j}^{<}, \hat{g}_{l j}^{>}$correlation Green functions for electrons:

$$
\begin{aligned}
\hat{g}_{l j}^{c, a}\left(1,1^{\prime} ; t_{0}\right) & =(\mathrm{i} \hbar)^{-1}\left\langle T^{c, a}\left[\hat{\psi}_{l H}(1), \hat{\psi}_{j H}^{+}\left(1^{\prime}\right)\right]\right\rangle^{t_{0}}, \\
\hat{g}_{l j}^{>}\left(1,1^{\prime} ; t_{0}\right) & =(\mathrm{i} \hbar)^{-1}\left\langle\hat{\psi}_{l H}(1) \hat{\psi}_{j H}^{+}\left(1^{\prime}\right)\right\rangle^{t_{0}} \\
\hat{g}_{j l}^{<}\left(1,1^{\prime} ; t_{0}\right) & =-(\mathrm{i} \hbar)^{-1}\left\langle\hat{\psi}_{j H}^{+}\left(1^{\prime}\right) \hat{\psi}_{l H}(1)\right\rangle^{t_{0}},
\end{aligned}
$$

where $(1)=\left(\mathbf{r}_{1}, \mathbf{s}_{1}, t_{1}\right),\left(1^{\prime}\right)=\left(\mathbf{r}_{1}^{\prime}, \mathbf{s}_{1}^{\prime}, t_{1}^{\prime}\right) \cdot \hat{\psi}_{l H}(1), \hat{\psi}_{j H}^{+}\left(1^{\prime}\right)$ are field operators of electrons in Heisenberg representation

$$
\hat{\psi}_{l H}(1)=U\left(t_{0}, t\right) \hat{\psi}_{l}\left(\mathbf{r}_{1}, \mathbf{s}_{1}\right) U\left(t, t_{0}\right), \quad U\left(t, t_{0}\right)=\mathrm{e}^{-\mathrm{i} / \hbar\left(t-t_{0}\right) H} .
$$

$T^{c, a}$ are direct and reverse time ordering operators. $\hat{g}_{l j}^{c}, \hat{g}_{l j}^{a}, \hat{g}_{l j}^{<}, \hat{g}_{l j}^{>}$define retarded and advanced Green functions $\hat{g}_{l j}^{R}, \hat{g}_{l j}^{A}$, by the relations

$$
\begin{aligned}
& \hat{g}_{l j}^{R}=\hat{g}_{l j}^{c}-\hat{g}_{l j}^{<}=\hat{g}_{l j}^{>}-\hat{g}_{l j}^{a}, \\
& \hat{g}_{l j}^{A}=\hat{g}_{l j}^{c}-\hat{g}_{l j}^{>}=\hat{g}_{l j}^{<}-\hat{g}_{l j}^{a} .
\end{aligned}
$$

The functions

$$
\hat{G}_{l j}\left(1,1^{\prime} ; t_{0}\right)=(\mathrm{i} \hbar)^{-1}\left\langle T_{C}\left[\hat{\psi}_{l H}(1), \hat{\psi}_{j H}^{+}\left(1^{\prime}\right)\right]\right\rangle^{t_{0}},
$$

satisfy the equation of Dyson type in Keldysh formalism [12,13,21,22]. $T_{C}$ is an operator of time ordering on a Keldysh contour $C$ [21]. Calculation of averages $\langle\ldots\rangle^{t_{0}}$ in Green functions is made using a nonequilibrium statistical operator $\left.\rho(t)\right|_{t=t_{0}}$ in the initial time which should be defined from the solution of the quantum Liouville equation of our system "metal-adsorbate-gas". Problems of averaging on the initial nonequilibrium states in Green functions have been analysed in detail in papers $[13,21,22]$, where a mixed Green functions formalism as a generalization of the Keldysh-Schwinger formalism is proposed. Such an approach could make it possible in our case to take into account the influence of diffusion-reaction gas processes 
on a surface on electron processes via averaging in the corresponding Green function using the nonequilibrium statistical operator of gas subsystem in initial time. In particular, it can be shown based on [22], that the correlation Green function $\hat{g}_{j l}^{<}\left(1,1^{\prime} ; t_{0}\right)$ in the limit $t_{0} \rightarrow-\infty$ and $t_{1}=t_{1}^{\prime}$ is equal to one-particle density matrix in $r$-representation

$$
f_{l j}\left(\mathbf{r}_{1}, \mathbf{s}_{1}, \mathbf{r}_{1}^{\prime}, \mathbf{s}_{1}^{\prime}, t_{1}\right)=-\left.\mathrm{i} \hbar \lim _{t_{0} \rightarrow-\infty} \hat{g}_{j l}^{<}\left(1,1^{\prime} ; t_{0}\right)\right|_{t_{1}=t_{1}^{\prime}} .
$$

It gives the connection of $\hat{g}_{j l}^{<}\left(1,1^{\prime} ; t_{0}\right)$ with the electron current.

For a consistent description of both electron kinetic and diffusion-reaction processes of adsorbed and nonadsorbed gas atoms in a system "metal-adsorbate-gas" we use the method of nonequilibrium statistical operator (NSO) by D.N.Zubarev [12]. This method is based on Bogolubov's ideas of a shortened description of nonequilibrium state of a system using the set of observed parameters. Such parameters of the shortened description can be nonequilibrium mean values of the electron subsystem:

$$
\left\langle\hat{A}_{j \nu \sigma}^{+\alpha} \hat{A}_{l \xi \sigma^{\prime}}^{\beta}\right\rangle^{t}=\operatorname{Sp}\left(\hat{A}_{j \nu \sigma}^{+\alpha} \hat{A}_{l \xi \sigma^{\prime}}^{\beta} \rho(t)\right)
$$

is the nonequilibrium one-electron density matrix; the mean densities of adsorbed and non-adsorbed gas atoms or molecules on a metal surface are:

$$
\left\langle\hat{n}_{\bar{a}}^{\nu}(\mathbf{R})\right\rangle^{t}=\operatorname{Sp}\left(\hat{n}_{\bar{a}}^{\nu}(\mathbf{R}) \rho(t)\right), \quad\left\langle\hat{n}_{a}(\mathbf{r})\right\rangle^{t}=\operatorname{Sp}\left(\hat{n}_{a}(\mathbf{r}) \rho(t)\right),
$$

and

$$
\left\langle\hat{\mathbf{d}}_{a}(\mathbf{r})\right\rangle^{t}=\operatorname{Sp}\left(\hat{\mathbf{d}}_{a}(\mathbf{r}) \rho(t)\right)
$$

is the mean polarization of density of gas atoms or molecules,

$$
\hat{\mathbf{d}}_{a}(\mathbf{r})=\sum_{j=1}^{N_{a}} \mathbf{d}_{j} \delta\left(\mathbf{r}-\mathbf{r}_{j}\right)
$$

is the microscopic polarization of density of gas atoms or molecules, $\mathbf{d}_{j}$ is dipole moment of particle $j$;

$$
\left\langle\hat{G}_{\bar{a} \bar{b}}^{\nu, \mu}\left(\mathbf{R}, \mathbf{R}^{\prime}\right)\right\rangle^{t}=\operatorname{Sp}\left(\hat{G}_{\bar{a} \bar{b}}^{\nu, \mu}\left(\mathbf{R}, \mathbf{R}^{\prime}\right) \rho(t)\right)
$$

is the nonequilibrium pair of the distribution function of adsorbed atoms or molecules on the metal surface. Here $\hat{n}_{\bar{a}}^{\nu}(\mathbf{R})$ is the density operator of gas atoms which are adsorbed in a $\nu$-state on the metal surface;

$$
\hat{n}_{\bar{a}}^{\nu}(\mathbf{R})=\sum_{j}^{N_{a}^{a d}} \hat{\psi}_{\nu j}^{+}(\mathbf{R}) \hat{\psi}_{\nu j}(\mathbf{R})
$$

$\hat{\psi}_{\nu j}^{+}(\mathbf{R}), \hat{\psi}_{\nu j}(\mathbf{R})$ are the creation and the annihilation operators in a $\nu$-state of the adsorbed gas atoms on a metal surface.

$$
\hat{G}_{\bar{a} \bar{b}}^{\nu, \mu}\left(\mathbf{R}, \mathbf{R}^{\prime}\right)=\hat{n}_{\bar{a}}^{\nu}(\mathbf{R}) \hat{n}_{\bar{b}}^{\mu}\left(\mathbf{R}^{\prime}\right), \hat{n}_{a}(\mathbf{r})=\sum_{j=1}^{N_{a}} \delta\left(\mathbf{r}-\mathbf{r}_{j}\right)
$$


is the microscopic density of gas atoms or molecules. If the chemical bond, stimulated by the metal surface between the adsorbed atoms appears, then the coordinate of molecule (cluster), consisting of the two atoms in states $\mu$ and $\nu$, can be found, with the help of a transition from individual reference systems for each atom $\hat{n}_{\bar{a}}^{\nu}(\mathbf{R})$, $\hat{n}_{\bar{b}}^{\mu}\left(\mathbf{R}^{\prime}\right)$ to their mass center reference system. Then $\left\langle\hat{G}_{\bar{a} \bar{b}}^{\nu, \mu}\left(\mathbf{R}, \mathbf{R}^{\prime}\right)\right\rangle^{t}$ is a mean density of molecules, created in the chemical reaction between the adsorbed atoms on the metal surface. On the contrary, molecules, consisting of two atoms in states $\mu$ and $\nu$, under the influence of nonhomogeneus magnetic field, can at first dissociate into atoms and then be adsorbed by the metal surface. In this case, $\left\langle\hat{G}_{\bar{a} \bar{b}}^{\nu, \mu}\left(\mathbf{R}, \mathbf{R}^{\prime}\right)\right\rangle^{t}$ is the nonequilibrium quantum distribution function of the atoms on metal a surface. Mean values of parameters of shortened description are calculated using $\rho(t)$-NSO of electrons and atoms of our system. This operator satisfies the Liouville equation

$$
\frac{\partial}{\partial t} \rho(t)+\mathrm{i} L_{N} \rho(t)=0
$$

where $\mathrm{i} L_{N}$ is the Liouville operator which corresponds to the total Hamiltonian (2.1). One can distinguish in the structure of the operator i $L_{N}$ some classical and some quantum parts:

$$
\begin{aligned}
\mathrm{i} L_{N}= & \mathrm{i} L_{N}^{\mathrm{cl}}+\mathrm{i} L_{N}^{\mathrm{qun}}, \\
\mathrm{i} L_{N}^{\mathrm{cl}}= & \sum_{j=1}^{N_{a}} \frac{\mathbf{p}_{j}}{m_{a}} \frac{\partial}{\partial \mathbf{r}_{j}}-\frac{1}{2} \sum_{j \neq j^{\prime}}^{N_{a}} \frac{\partial}{\partial \mathbf{r}_{j}} V_{a a}\left(\left|\mathbf{r}_{j}-\mathbf{r}_{j^{\prime}}\right|\right)\left(\frac{\partial}{\partial \mathbf{p}_{j}}-\frac{\partial}{\partial \mathbf{p}_{j^{\prime}}}\right) \\
& -\sum_{j, \beta, f}^{N_{a}, N_{\beta}} \frac{\partial}{\partial \mathbf{r}_{j}}\left(V_{a \beta}\left(\mathbf{r}_{j}, \mathbf{R}_{f}\right)+U_{a}\left(z_{j}\right)\right) \frac{\partial}{\partial \mathbf{p}_{j}}
\end{aligned}
$$

is the classical part that corresponds to an interacting gas. $V_{a \beta}\left(\mathbf{r}_{j}, \mathbf{R}_{f}\right)$ are interaction potentials of gas atoms with other atoms of a system.

$$
\mathrm{i} \hat{L}_{N}^{\text {qun }} \hat{A}=\frac{1}{\mathrm{i} \hbar}\left[\hat{A}, H_{e}+H_{i}^{\mathrm{int}}+H_{a}^{\mathrm{int}}+U+H_{\text {reac }}\right]
$$

is the quantum part of the total Liouville operator. The nonequilibrium statistical operator of electrons, atoms of a "metal-adsorbate-gas" is normalized as

$$
\operatorname{Sp} \rho(t)=1
$$

where

$$
\operatorname{Sp}(\ldots)=\prod_{\alpha} \int \frac{(\mathrm{d} x)^{N_{\alpha}}}{N_{\alpha} !(2 \pi \hbar)^{3 N_{\alpha}}} \operatorname{Sp}_{(\nu, \xi, \sigma)}(\ldots), \quad \mathrm{d} x=\mathrm{d} \mathbf{r} \mathrm{d} \mathbf{p}, \quad N_{\alpha}=\left\{N_{a}, N_{\bar{a}}, N_{e}, N_{s}\right\},
$$

$\mathrm{Sp}_{(\nu, \xi, \sigma)}$ means summation over all values of spin and other quantum numbers. To find the nonequilibrium statistical operator $\rho(t)$ one needs a boundary condition. Using the NSO method by D.N.Zubarev $[12,18]$, we are looking for the solution of equation (2.9) in such a form, where time dependency is included indirectly via 
mean values of the set of the shortened description. To this end, let us introduce some infinitely small source into right hand side of the Liouville equation (2.9) which destroys its symmetry on time inversion and selects the needed retarded solutions $[12,18]$. Thus, we start further from the equation

$$
\left(\frac{\partial}{\partial t}+\mathrm{i} L_{N}\right) \rho(t)=-\varepsilon\left(\rho(t)-\rho_{q}(t)\right)
$$

where $\varepsilon \rightarrow+0$ after the thermodynamic limits transition. The auxiliary relevant statistical operator $\rho_{q}(t)$ is defined from the condition of extremum of information entropy of a system and conservation of normalization $\operatorname{Sp} \rho_{q}(t)=1$ and fixed values of parameters of the shortened description. In our case, these parameters are defined by relations $(2.5)-(2.8)$. Then, proceeding in a standard way $[12,18]$, one obtains the expression for a relevant statistical operator:

$$
\begin{aligned}
\rho_{q}(t)=\exp \{ & -\Phi(t)-\beta\left(H-\sum_{l, l^{\prime}} b\left(l, l^{\prime} ; t\right) \hat{N}_{l l^{\prime}}-\sum_{a} \int \mathrm{d} \mathbf{r} \mu_{a}(\mathbf{r} ; t) \hat{n}_{a}(\mathbf{r})\right. \\
& -\sum_{\bar{a}} \sum_{\nu} \int \mathrm{d} \mathbf{R} \mu_{\bar{a}}^{\nu}(\mathbf{R} ; t) \hat{n}_{\bar{a}}^{\nu}(\mathbf{R})-\sum_{a} \int \mathrm{d} \mathbf{r e}(\mathbf{r} ; t) \hat{\mathbf{d}}_{a}(\mathbf{r}) \\
& \left.\left.-\sum_{\bar{a} \bar{b}} \sum_{\nu \mu} \int \mathrm{d} \mathbf{R} \mathrm{d} \mathbf{R}^{\prime} M_{\bar{a} \bar{b}}^{\nu, \mu}\left(\mathbf{R}, \mathbf{R}^{\prime} ; t\right) \hat{G}_{\bar{a}}^{\nu, \mu}\left(\mathbf{R}, \mathbf{R}^{\prime}\right)\right)\right\}
\end{aligned}
$$

where

$$
\begin{aligned}
\Phi(t)=\ln \operatorname{Sp} \exp \{ & -\beta\left(H-\sum_{l, l^{\prime}} b\left(l, l^{\prime} ; t\right) \hat{N}_{l l^{\prime}}-\sum_{a} \int d \mathbf{r} \mu_{a}(\mathbf{r} ; t) \hat{n}_{a}(\mathbf{r})\right. \\
& -\sum_{\bar{a}} \sum_{\nu} \int d \mathbf{R} \mu_{\bar{a}}^{\nu}(\mathbf{R} ; t) \hat{n}_{\bar{a}}^{\nu}(\mathbf{R})-\sum_{a} \int d \mathbf{r e}(\mathbf{r} ; t) \hat{\mathbf{d}}_{a}(\mathbf{r}) \\
& \left.\left.-\sum_{\bar{a} \bar{b}} \sum_{\nu \mu} \int d \mathbf{R} d \mathbf{R}^{\prime} M_{\bar{a} \bar{b}}^{\nu, \mu}\left(\mathbf{R}, \mathbf{R}^{\prime} ; t\right) \hat{G}_{\bar{a} \bar{b}}^{\nu, \mu}\left(\mathbf{R}, \mathbf{R}^{\prime}\right)\right)\right\},
\end{aligned}
$$

is the Massieu-Planck functional. It is defined from the normalization condition of $\rho_{q}(t)$. The parameters $b\left(l, l^{\prime} ; t\right), \mu_{a}(\mathbf{r} ; t), \mu_{\bar{a}}^{\nu}(\mathbf{R} ; t), \mathbf{e}(\mathbf{r} ; t), M_{\bar{a} \bar{b}}^{\nu, \mu}\left(\mathbf{R}, \mathbf{R}^{\prime} ; t\right)$ are defined from the self-consistency conditions

$$
\begin{aligned}
& \left\langle\hat{N}_{l l^{\prime}}\right\rangle^{t}=\left\langle\hat{N}_{l l^{\prime}}\right\rangle_{q}^{t}, \quad\left\langle\hat{n}_{a}(\mathbf{r})\right\rangle^{t}=\left\langle\hat{n}_{a}(\mathbf{r})\right\rangle_{q}^{t}, \\
& \left\langle\hat{n}_{\bar{a}}^{\nu}(\mathbf{R})\right\rangle^{t}=\left\langle\hat{n}_{\bar{a}}^{\nu}(\mathbf{R})\right\rangle_{q}^{t}, \quad\left\langle\hat{\mathbf{d}}_{a}(\mathbf{r})\right\rangle^{t}=\left\langle\hat{\mathbf{d}}_{a}(\mathbf{r})\right\rangle_{q}^{t}, \\
& \left\langle\hat{G}_{\bar{a} \bar{b}}^{\nu, \mu}\left(\mathbf{R}, \mathbf{R}^{\prime}\right)\right\rangle^{t}=\left\langle\hat{G}_{\bar{a} \bar{b}}^{\nu, \mu}\left(\mathbf{R}, \mathbf{R}^{\prime}\right)\right\rangle_{q}^{t},
\end{aligned}
$$

and denote that $\mu_{a}(\mathbf{r} ; t)$ is the local chemical potential of a gas atom; $\mu_{\bar{a}}^{\nu}(\mathbf{R} ; t)$ is the local chemical potential of an adsorbed atom in a state $\nu$ on a metal surface; $\mathbf{e}(\mathbf{r} ; t)$ is the local electric field, which is made up of the electron and ion subsystem on metal surface, and is defined by the Maxwellian equation:

$$
\nabla \mathbf{e}(\mathbf{R} ; t)=4 \pi\left(\left\langle\hat{\rho}_{e}(\mathbf{R})\right\rangle^{t}+\left\langle\sum_{f} Z_{f} e \hat{n}_{f}(\mathbf{R})\right\rangle^{t}\right)
$$


where

$$
\hat{n}_{e}(\mathbf{R})=\sum_{\mathbf{s}} \hat{\psi}^{+}(\mathbf{R}, \mathbf{s}) \hat{\psi}(\mathbf{R}, \mathbf{s})
$$

is the density operator of the electron subsystem on a metal surface, $\hat{\rho}_{e}(\mathbf{R})=e \hat{n}_{e}(\mathbf{R})$ is the density operator charge of electrons, e is the charge of electron, and

$$
\hat{n}_{f}(\mathbf{R})=\sum_{\mathbf{s}^{\prime}} \hat{\psi}_{f}^{+}\left(\mathbf{R}, \mathbf{s}^{\prime}\right) \hat{\psi}_{f}\left(\mathbf{R}, \mathbf{s}^{\prime}\right)
$$

is the density operator of the ion subsystem on the metal surface, $\mathbf{s}^{\prime}$ is a spin coordinate and $Z_{f}$ is the valence of ion on metal surface. $M_{\bar{a} \bar{b}}^{\nu, \mu}\left(\mathbf{R}, \mathbf{R}^{\prime} ; t\right)$ is the local chemical potential of adsorbed complex atoms $\bar{a}, \bar{b}$ in the states $\nu$ and $\mu$ on a metal surface; $\beta=1 / k_{\mathrm{B}} T, k_{\mathrm{B}}$ is the Boltzmann constant, $T$ is the equilibrium value of temperature. Here $\hat{N}_{l l^{\prime}}=\hat{A}_{l}^{+} \hat{A}_{l^{\prime}}, l, l^{\prime}$ indicate the set of indices $\{\alpha, j \nu \sigma\} ;\langle(\ldots)\rangle_{q}^{t}$ $=\operatorname{Sp}(\ldots) \rho_{q}(t)$. Using the standard NSO procedure with taking account the projection $[12,18]$ and structure of $\rho_{q}(t)(2.11)$ one obtains from $(2.10)$ the expression for the nonequilibrium statistical operator:

$$
\begin{aligned}
\rho(t)= & \rho_{q}(t)+\sum_{l l^{\prime}} \int_{-\infty}^{t} \mathrm{e}^{\epsilon\left(t^{\prime}-t\right)} T\left(t, t^{\prime}\right) \int_{0}^{1} \mathrm{~d} \tau \rho_{q}^{\tau}\left(t^{\prime}\right) I_{N}\left(l, l^{\prime} ; t^{\prime}\right) \rho_{q}^{1-\tau}\left(t^{\prime}\right) \beta b\left(l, l^{\prime} ; t^{\prime}\right) \mathrm{d} t^{\prime} \\
& +\sum_{a} \int_{-\infty} \mathrm{d} \mathbf{r} \int_{-\infty}^{t} \mathrm{e}^{\epsilon\left(t^{\prime}-t\right)} T\left(t, t^{\prime}\right) \int_{0}^{1} \mathrm{~d} \tau \rho_{q}^{\tau}\left(t^{\prime}\right) I_{a}\left(\mathbf{r} ; t^{\prime}\right) \rho_{q}^{1-\tau}\left(t^{\prime}\right) \beta \mu_{a}\left(\mathbf{r} ; t^{\prime}\right) \mathrm{d} t^{\prime} \\
& +\sum_{\bar{a}} \sum_{\nu} \int_{-\infty} \mathrm{d} \mathbf{R} \int_{-\infty}^{t} \mathrm{e}^{\epsilon\left(t^{\prime}-t\right)} T\left(t, t^{\prime}\right) \int_{0}^{1} \mathrm{~d} \tau \rho_{q}^{\tau}\left(t^{\prime}\right) I_{\bar{a}}^{\nu}\left(\mathbf{R} ; t^{\prime}\right) \rho_{q}^{1-\tau}\left(t^{\prime}\right) \beta \mu_{\bar{a}}^{\nu}\left(\mathbf{R} ; t^{\prime}\right) \mathrm{d} t^{\prime} \\
& +\sum_{a} \int_{-\infty} \mathrm{d} \mathbf{r} \int_{-\infty}^{\epsilon} \mathrm{e}^{\epsilon\left(t^{\prime}-t\right)} T\left(t, t^{\prime}\right) \int_{0}^{1} \mathrm{~d} \tau \rho_{q}^{\tau}\left(t^{\prime}\right) I_{d}^{a}\left(\mathbf{r} ; t^{\prime}\right) \rho_{q}^{1-\tau}\left(t^{\prime}\right) \beta \mathbf{e}\left(\mathbf{r} ; t^{\prime}\right) \mathrm{d} t^{\prime} \\
& +\sum_{\bar{a}, \bar{b}} \sum_{\nu, \mu} \int^{t} \mathrm{~d} \mathbf{R} \int^{1} \mathrm{~d} \mathbf{R}^{\prime} \int_{-\infty}^{\epsilon\left(t^{\prime}-t\right)} T\left(t, t^{\prime}\right) \\
& \times \int_{0} \mathrm{~d} \tau \rho_{q}^{\tau}\left(t^{\prime}\right) I_{G_{\bar{a} \bar{b}} \mu}^{\nu \mu}\left(\mathbf{R} \mathbf{R}^{\prime} ; t^{\prime}\right) \rho_{q}^{1-\tau}\left(t^{\prime}\right) \beta M_{\bar{a} \bar{b}}^{\nu \mu}\left(\mathbf{R R}^{\prime} ; t^{\prime}\right) \mathrm{d} t^{\prime},
\end{aligned}
$$

where

$$
T\left(t, t^{\prime}\right)=\exp \left\{-\int_{t^{\prime}}^{t}\left(1-\mathcal{P}_{q}\left(t^{\prime \prime}\right)\right) \mathrm{i} L_{N} \mathrm{~d} t^{\prime \prime}\right\}
$$

is an evolution operator taking into account projection. $\mathcal{P}_{q}(t)$ is the KawasakiGunton projection operator. It acts on the statistical operator and has got the 
properties like this:

$$
\mathcal{P}_{q}(t) \rho\left(t^{\prime}\right)=\rho_{q}(t), \quad \mathcal{P}_{q}(t) \rho_{q}\left(t^{\prime}\right)=\rho_{q}(t), \quad \mathcal{P}_{q}(t) \mathcal{P}_{q}\left(t^{\prime}\right)=\mathcal{P}_{q}(t) .
$$

It is connected with the Mori projection operator $\mathcal{P}(t)$ by the relation:

$$
\begin{aligned}
\mathcal{P}_{q}(t) \hat{A} \rho_{q}(t)= & \int_{0}^{1} \mathrm{~d} \tau\left(\rho_{q}\right)^{\tau} \mathcal{P}(t) \hat{A} \rho_{q}^{\prime}(t)^{1-\tau}, \\
\mathcal{P}(t) \hat{A}= & \langle\hat{A}\rangle_{q}^{t}+\sum_{l, l^{\prime}} \frac{\delta\langle\hat{A}\rangle_{q}^{t}}{\delta\left\langle\hat{N}_{l l^{\prime}}\right\rangle^{t}}\left(\hat{N}_{l l^{\prime}}-\left\langle\hat{N}_{l l^{\prime}}\right\rangle^{t}\right) \\
& +\sum_{a} \int_{\mathrm{a}} \mathrm{d} \mathbf{r} \frac{\delta\langle\hat{A}\rangle_{q}^{t}}{\delta\left\langle\hat{n}_{a}(\mathbf{r})\right\rangle^{t}}\left(\hat{n}_{a}(\mathbf{r})-\left\langle\hat{n}_{a}(\mathbf{r})\right\rangle^{t}\right) \\
& +\sum_{\bar{a}} \sum_{\nu} \int \mathrm{d} \mathbf{R} \frac{\delta\langle\hat{A}\rangle_{q}^{t}}{\delta\left\langle\hat{n}_{\bar{a}}^{\nu}(\mathbf{R})\right\rangle^{t}}\left(\hat{n}_{\bar{a}}^{\nu}(\mathbf{R})-\left\langle\hat{n}_{\bar{a}}^{\nu}(\mathbf{R})\right\rangle^{t}\right) . \\
& +\sum_{a} \int \mathrm{d} \mathbf{r} \frac{\delta\langle\hat{A}\rangle_{q}^{t}}{\delta\left\langle\hat{\mathbf{d}}_{a}(\mathbf{r})\right\rangle^{t}}\left(\hat{\mathbf{d}}_{a}(\mathbf{r})-\left\langle\hat{\mathbf{d}}_{a}(\mathbf{r})\right\rangle^{t}\right) \\
& +\sum_{\bar{a} \bar{b}} \sum_{\nu \mu} \int \mathrm{d} \mathbf{R} \int \mathrm{d} \mathbf{R}^{\prime} \\
& \times \frac{\delta\langle\hat{A}\rangle_{q}^{t}}{\delta\left\langle\hat{G}_{\bar{a} \bar{b}}^{\nu, \mu}\left(\mathbf{R}, \mathbf{R}^{\prime}\right)\right\rangle^{t}}\left(\hat{G}_{\bar{a} \bar{b}}^{\nu, \mu}\left(\mathbf{R}, \mathbf{R}^{\prime}\right)-\left\langle\hat{G}_{\bar{a} \bar{b}}^{\nu, \mu}\left(\mathbf{R}, \mathbf{R}^{\prime}\right)\right\rangle^{t}\right) .
\end{aligned}
$$

$\mathcal{P}(t)$ acts on the operators and has got the properties of a projection operator:

$$
\begin{aligned}
& \mathcal{P}(t) \hat{n}_{a}(\mathbf{r})=\hat{n}_{a}(\mathbf{r}), \quad \mathcal{P}(t) \hat{N}_{l l^{\prime}}=\hat{N}_{l l^{\prime}}, \\
& \mathcal{P}(t) \hat{n}_{\bar{a}}(\mathbf{R})=\hat{n}_{\bar{a}}(\mathbf{R}), \quad \mathcal{P}(t) \hat{\mathbf{d}}_{a}(\mathbf{r})=\hat{\mathbf{d}}_{a}(\mathbf{r}), \\
& \mathcal{P}(t) \hat{G}_{\bar{a} \bar{b}}^{\nu, \mu}\left(\mathbf{R}, \mathbf{R}^{\prime}\right)=\hat{G}_{\bar{a} \bar{b}}^{\nu, \mu}\left(\mathbf{R}, \mathbf{R}^{\prime}\right), \\
& \mathcal{P}(t) \mathcal{P}\left(t^{\prime}\right)=\mathcal{P}(t), \quad \mathcal{P}(t)(1-\mathcal{P}(t))=0 ; \\
& I_{N}\left(l, l^{\prime} ; t^{\prime}\right)=\left(1-\mathcal{P}\left(t^{\prime}\right)\right) \dot{\hat{\hat{N}}}_{l l^{\prime}}, \quad I_{a}\left(\mathbf{r} ; t^{\prime}\right)=\left(1-\mathcal{P}\left(t^{\prime}\right)\right) \dot{\hat{n}}_{a}(\mathbf{r}), \\
& I_{\bar{a}}^{\nu}\left(\mathbf{R} ; t^{\prime}\right)=\left(1-\mathcal{P}\left(t^{\prime}\right)\right) \hat{\hat{n}}_{\bar{a}}^{\nu}(\mathbf{R}), \\
& I_{d}^{a}\left(\mathbf{r} ; t^{\prime}\right)=\left(1-\mathcal{P}\left(t^{\prime}\right)\right) \dot{\hat{\mathbf{d}}}_{a}(\mathbf{r}), \\
& I_{G_{\bar{a} \bar{b}}}^{\nu \mu}\left(\mathbf{R R}^{\prime} ; t^{\prime}\right)=\left(1-\mathcal{P}\left(t^{\prime}\right)\right) \dot{\hat{G}}_{\bar{a} \bar{b}}^{\nu, \mu}\left(\mathbf{R}, \mathbf{R}^{\prime}\right)
\end{aligned}
$$

are generalized flows;

$$
\begin{aligned}
& \dot{\hat{N}}_{l l^{\prime}}=\frac{1}{i \hbar}\left[\hat{N}_{l l^{\prime}}, H\right], \quad \dot{\hat{n}}_{\bar{a}}^{\nu}(\mathbf{R})=\frac{1}{\mathrm{i} \hbar}\left[\hat{n}_{\bar{a}}^{\nu}(\mathbf{R}), H\right], \\
& \dot{\hat{n}}_{a}(\mathbf{r})=\mathrm{i} L_{N}^{\mathrm{cl}} \hat{n}_{a}(\mathbf{r})=\frac{1}{m_{a}} \boldsymbol{\nabla} \cdot \hat{\mathbf{p}}_{a}(\mathbf{r}), \\
& \hat{\mathbf{p}}_{a}(\mathbf{r})=\sum_{j=1}^{N_{a}} \mathbf{p}_{j} \delta\left(\mathbf{r}-\mathbf{r}_{j}\right)
\end{aligned}
$$


is the microscopic momentum density of gas atoms;

$$
\dot{\hat{\mathbf{d}}}_{a}(\mathbf{r})=\mathrm{i} L_{N}^{\mathrm{cl}} \hat{\mathbf{d}}_{a}(\mathbf{r}), \quad \dot{\hat{G}}_{\bar{a} \bar{b}}^{\nu, \mu}\left(\mathbf{R}, \mathbf{R}^{\prime}\right)=\frac{1}{\mathrm{i} \hbar}\left[\hat{G}_{\bar{a} \bar{b}}^{\nu, \mu}\left(\mathbf{R}, \mathbf{R}^{\prime}\right), H\right]
$$

In such a way, we obtained a general expression for the nonequilibrium statistical operator $\rho(t)$ of electrons and gas atoms in a system "metal-adsorbate-gas" for the specific set of parameters of shortened description (2.5)-(2.8). It depends on generalized flows (2.14) which describe dissipative transport processes in a system. As far as due to a shortened description $\rho(t)$ is known to be a functional of parameters $\left\langle\hat{N}_{l l^{\prime}}\right\rangle^{t},\left\langle\hat{n}_{a}(\mathbf{r})\right\rangle^{t},\left\langle\hat{n}_{\bar{a}}^{\nu}(\mathbf{R})\right\rangle^{t},\left\langle\hat{\mathbf{d}}_{a}(\mathbf{r})\right\rangle^{t}$, into $\left\langle\hat{G}_{\bar{a} \bar{b}}^{\nu, \mu}\left(\mathbf{R}, \mathbf{R}^{\prime}\right)\right\rangle^{t}$, we can start from the explicit expression for $\rho_{q}(t)$ taking into account the self-consistency conditions (2.12). To obtain them let us use the identity:

$$
\frac{\partial}{\partial t}\left\langle\hat{B}_{n}\right\rangle^{t}=\left\langle\mathrm{i} L_{N} \hat{B}_{n}\right\rangle^{t}=\left\langle\mathrm{i} L_{N} \hat{B}_{n}\right\rangle_{q}^{t}+\left\langle I_{B}(t)\right\rangle^{t}
$$

where $\hat{B}_{n}=\left(\hat{N}_{l l^{\prime}}, \hat{n}_{a}(\mathbf{r}), \hat{n}_{\bar{a}}^{\nu}(\mathbf{R}), \hat{\mathbf{d}}_{a}(\mathbf{r}), \hat{G}_{\bar{a} \bar{b}}^{\nu, \mu}\left(\mathbf{R}, \mathbf{R}^{\prime}\right)\right)$ and

$$
I_{B}(t)=\left(I_{N}\left(l, l^{\prime} ; t\right), I_{a}(\mathbf{r} ; t), I_{\bar{a}}^{\nu}(\mathbf{R} ; t), I_{d}^{a}\left(\mathbf{r} ; t^{\prime}\right), I_{G_{\bar{a} \bar{b}}}^{\nu \mu}\left(\mathbf{R} \mathbf{R}^{\prime} ; t^{\prime}\right) .\right.
$$

Averaging the right hand parts of these equalities using NSO (2.14) one obtains generalized transport equations for one-electron density matrix and mean values of densities of adsorbed and non-adsorbed gas atoms:

$$
\begin{aligned}
& \frac{\partial}{\partial t}\left\langle\hat{N}_{l l^{\prime}}\right\rangle^{t}=\left\langle\dot{\hat{N}}_{l l^{\prime}}\right\rangle_{q}^{t}+\sum_{j, j^{\prime}} \int_{-\infty}^{t} \mathrm{e}^{\epsilon\left(t^{\prime}-t\right)} \varphi_{N N}\left(l l^{\prime}, j j^{\prime} ; t, t^{\prime}\right) \beta b\left(j, j^{\prime} ; t^{\prime}\right) \mathrm{d} t^{\prime} \\
& +\sum_{a} \int \mathrm{d} \mathbf{r}^{\prime} \int_{-\infty}^{t} \mathrm{e}^{\epsilon\left(t^{\prime}-t\right)} \varphi_{N n_{a}}\left(l l^{\prime}, \mathbf{r} ; t, t^{\prime}\right) \beta \mu_{a}\left(\mathbf{r}^{\prime} ; t^{\prime}\right) \mathrm{d} t^{\prime} \\
& +\sum_{\bar{a}} \sum_{\nu^{\prime}} \int \mathrm{d} \mathbf{R}^{\prime} \int_{-\infty}^{t} \mathrm{e}^{\epsilon\left(t^{\prime}-t\right)} \varphi_{N n_{\bar{a}}}^{\nu^{\prime}}\left(l l^{\prime}, \mathbf{R} ; t, t^{\prime}\right) \beta \mu_{\bar{a}}^{\nu^{\prime}}\left(\mathbf{R}^{\prime} ; t^{\prime}\right) \mathrm{d} t^{\prime} \\
& +\sum_{a} \int \mathrm{d} \mathbf{r}^{\prime} \int_{-\infty}^{t} \mathrm{e}^{\epsilon\left(t^{\prime}-t\right)} \varphi_{N d_{a}}\left(l l^{\prime}, \mathbf{r}^{\prime} ; t, t^{\prime}\right) \beta \mathbf{e}\left(\mathbf{r}^{\prime} ; t^{\prime}\right) \mathrm{d} t^{\prime} \\
& +\sum_{\bar{a} \bar{b}} \sum_{\nu^{\prime} \mu^{\prime}} \int \mathrm{d} \mathbf{R}^{\prime} \int \mathrm{d} \mathbf{R}^{\prime \prime} \\
& \times \int_{-\infty}^{t} \mathrm{e}^{\epsilon\left(t^{\prime}-t\right)} \varphi_{N G_{\bar{a} \bar{b}} \nu^{\prime} \mu^{\prime}}\left(l l^{\prime}, \mathbf{R}^{\prime} \mathbf{R}^{\prime \prime} ; t, t^{\prime}\right) \beta M_{\bar{a} \bar{b}}^{\nu^{\prime} \mu^{\prime}}\left(\mathbf{R}^{\prime} \mathbf{R}^{\prime \prime} ; t^{\prime}\right) \mathrm{d} t^{\prime} \\
& \frac{\partial}{\partial t}\left\langle\hat{n}_{a}(\mathbf{r})\right\rangle^{t}=\left\langle\dot{\hat{n}}_{a}(\mathbf{r})\right\rangle_{q}^{t}+\sum_{j, j^{\prime}} \int_{-\infty}^{t} \mathrm{e}^{\epsilon\left(t^{\prime}-t\right)} \varphi_{n_{a}, N}\left(\mathbf{r} ; j, j^{\prime} ; t, t^{\prime}\right) \beta b\left(j, j^{\prime} ; t^{\prime}\right) \mathrm{d} t^{\prime}
\end{aligned}
$$




$$
\begin{aligned}
& +\sum_{b} \int \mathrm{d} \mathbf{r}^{\prime} \int_{-\infty}^{t} \mathrm{e}^{\epsilon\left(t^{\prime}-t\right)} \varphi_{n_{a} n_{b}}\left(\mathbf{r}, \mathbf{r}^{\prime} ; t, t^{\prime}\right) \beta \mu_{b}\left(\mathbf{r}^{\prime} ; t^{\prime}\right) \mathrm{d} t^{\prime} \\
& +\sum_{\bar{b}} \sum_{\nu^{\prime}} \int \mathrm{d} \mathbf{R}^{\prime} \int_{-\infty}^{t} \mathrm{e}^{\epsilon\left(t^{\prime}-t\right)} \varphi_{n_{n_{n}} \nu_{\bar{b}}^{\prime}}\left(\mathbf{r}, \mathbf{R}^{\prime} ; t, t^{\prime}\right) \beta \mu_{\bar{b}}^{\nu^{\prime}}\left(\mathbf{R}^{\prime} ; t^{\prime}\right) \mathrm{d} t^{\prime} \\
& +\sum_{b} \int \mathrm{d} \mathbf{r}^{\prime} \int_{-\infty}^{t} \mathrm{e}^{\epsilon\left(t^{\prime}-t\right)} \varphi_{n_{a} d_{b}}\left(\mathbf{r}, \mathbf{r}^{\prime} ; t, t^{\prime}\right) \beta \mathbf{e}\left(\mathbf{r}^{\prime} ; t^{\prime}\right) \mathrm{d} t^{\prime} \\
& +\sum_{\bar{a}^{\prime} \bar{b}} \sum_{\nu^{\prime} \mu^{\prime}} \int \mathrm{d} \mathbf{R}^{\prime} \int \mathrm{d} \mathbf{R}^{\prime \prime} \\
& \times \int_{-\infty}^{t} \mathrm{e}^{\epsilon\left(t^{\prime}-t\right)} \varphi_{n_{a} G_{\bar{a}^{\prime} \bar{b}}}^{\nu^{\prime} \mu^{\prime}}\left(\mathbf{r}, \mathbf{R}^{\prime} \mathbf{R}^{\prime \prime} ; t, t^{\prime}\right) \beta M_{\bar{a}^{\prime} \bar{b}}^{\nu^{\prime} \mu^{\prime}}\left(\mathbf{R}^{\prime} \mathbf{R}^{\prime \prime} ; t^{\prime}\right) \mathrm{d} t^{\prime} \\
& \frac{\partial}{\partial t}\left\langle\hat{n}_{\bar{a}}^{\nu}(\mathbf{R})\right\rangle^{t}=\left\langle\dot{\hat{n}}_{\bar{a}}^{\nu}(\mathbf{R})\right\rangle_{q}^{t}+\sum_{j, j^{\prime}} \int_{-\infty}^{t} \mathrm{e}^{\epsilon\left(t^{\prime}-t\right)} \varphi_{n_{\bar{a}}, N}\left(\mathbf{R} ; j, j^{\prime} ; t, t^{\prime}\right) \beta b\left(j, j^{\prime} ; t^{\prime}\right) \mathrm{d} t^{\prime} \\
& +\sum_{b} \int \mathrm{d} \mathbf{r}^{\prime} \int_{-\infty}^{t} \mathrm{e}^{\epsilon\left(t^{\prime}-t\right)} \varphi_{n_{\bar{a}} n_{b}}^{\nu}\left(\mathbf{R}, \mathbf{r}^{\prime} ; t, t^{\prime}\right) \beta \mu_{b}\left(\mathbf{r}^{\prime} ; t^{\prime}\right) \mathrm{d} t^{\prime} \\
& +\sum_{\bar{b}} \sum_{\nu^{\prime}} \int \mathrm{d} \mathbf{R}^{\prime} \int_{-\infty}^{t} \mathrm{e}^{\epsilon\left(t^{\prime}-t\right)} \varphi_{n_{\bar{a}} n_{\bar{b}}}^{\nu \nu^{\prime}}\left(\mathbf{R}, \mathbf{R}^{\prime} ; t, t^{\prime}\right) \beta \mu_{\bar{b}}^{\nu^{\prime}}\left(\mathbf{R}^{\prime} ; t^{\prime}\right) \mathrm{d} t^{\prime} \\
& +\sum_{b} \int \mathrm{d} \mathbf{r}^{\prime} \int_{-\infty}^{t} \mathrm{e}^{\epsilon\left(t^{\prime}-t\right)} \varphi_{n_{\bar{a}} d_{b}}\left(\mathbf{r}, \mathbf{r}^{\prime} ; t, t^{\prime}\right) \beta \mathbf{e}\left(\mathbf{r}^{\prime} ; t^{\prime}\right) \mathrm{d} t^{\prime} \\
& +\sum_{\bar{a}^{\prime} \bar{b}} \sum_{\nu^{\prime} \mu^{\prime}} \int \mathrm{d} \mathbf{R}^{\prime} \int \mathrm{d} \mathbf{R}^{\prime \prime} \\
& \times \int_{-\infty}^{t} \mathrm{e}^{\epsilon\left(t^{\prime}-t\right)} \varphi_{n_{\bar{a}} G_{\bar{a}}^{\prime} \bar{b}}^{\nu^{\prime} \mu^{\prime}}\left(\mathbf{r}, \mathbf{R}^{\prime} \mathbf{R}^{\prime \prime} ; t, t^{\prime}\right) \beta M_{\bar{a}^{\prime} \bar{b}}^{\nu^{\prime} \mu^{\prime}}\left(\mathbf{R}^{\prime} \mathbf{R}^{\prime \prime} ; t^{\prime}\right) \mathrm{d} t^{\prime} \\
& \frac{\partial}{\partial t}\left\langle\hat{\mathbf{d}}_{a}(\mathbf{r})\right\rangle^{t}=\left\langle\dot{\hat{\mathbf{d}}}_{a}(\mathbf{r})\right\rangle_{q}^{t}+\sum_{j, j^{\prime}} \int_{-\infty}^{t} \mathrm{e}^{\epsilon\left(t^{\prime}-t\right)} \varphi_{d_{a}, N}\left(\mathbf{r} ; j, j^{\prime} ; t, t^{\prime}\right) \beta b\left(j, j^{\prime} ; t^{\prime}\right) \mathrm{d} t^{\prime} \\
& +\sum_{b} \int \mathrm{d} \mathbf{r}^{\prime} \int_{-\infty}^{t} \mathrm{e}^{\epsilon\left(t^{\prime}-t\right)} \varphi_{d_{a} n_{b}}\left(\mathbf{r}, \mathbf{r}^{\prime} ; t, t^{\prime}\right) \beta \mu_{b}\left(\mathbf{r}^{\prime} ; t^{\prime}\right) \mathrm{d} t^{\prime}
\end{aligned}
$$




$$
\begin{aligned}
& +\sum_{\bar{b}} \sum_{\nu^{\prime}} \int \mathrm{d} \mathbf{R}^{\prime} \int_{-\infty}^{t} \mathrm{e}^{\epsilon\left(t^{\prime}-t\right)} \varphi_{d_{a} n_{\bar{b}}}^{\nu^{\prime}}\left(\mathbf{r}, \mathbf{R}^{\prime} ; t, t^{\prime}\right) \beta \mu_{\bar{b}}^{\nu^{\prime}}\left(\mathbf{R}^{\prime} ; t^{\prime}\right) \mathrm{d} t^{\prime} \\
& +\sum_{b} \int \mathrm{d} \mathbf{r}^{\prime} \int_{-\infty}^{t} \mathrm{e}^{\epsilon\left(t^{\prime}-t\right)} \varphi_{d_{a} d_{b}}\left(\mathbf{r}, \mathbf{r}^{\prime} ; t, t^{\prime}\right) \beta \mathbf{e}\left(\mathbf{r}^{\prime} ; t^{\prime}\right) \mathrm{d} t^{\prime} \\
& +\sum_{\bar{a}^{\prime} \bar{b}} \sum_{\nu^{\prime} \mu^{\prime}} \int \mathrm{d} \mathbf{R}^{\prime} \int \mathrm{d} \mathbf{R}^{\prime \prime} \\
& \times \int_{-\infty}^{t} \mathrm{e}^{\epsilon\left(t^{\prime}-t\right)} \varphi_{d_{a} G_{\bar{a}^{\prime} \bar{b}}}^{\nu^{\prime} \mu^{\prime}}\left(\mathbf{r}, \mathbf{R}^{\prime} \mathbf{R}^{\prime \prime} ; t, t^{\prime}\right) \beta M_{\bar{a}^{\prime} \bar{b}}^{\nu^{\prime} \mu^{\prime}}\left(\mathbf{R}^{\prime} \mathbf{R}^{\prime \prime} ; t^{\prime}\right) \mathrm{d} t^{\prime} \\
& \frac{\partial}{\partial t}\left\langle\hat{G}_{\bar{a} \bar{b}}^{\nu \mu}\left(\mathbf{R}, \mathbf{R}^{\prime}\right)\right\rangle^{t}=\left\langle\dot{\hat{G}}_{\bar{a} \bar{b}}^{\nu \mu}\left(\mathbf{R}, \mathbf{R}^{\prime}\right)\right\rangle_{q}^{t} \\
& +\sum_{j, j^{\prime}} \int_{-\infty}^{t} \mathrm{e}^{\epsilon\left(t^{\prime}-t\right)} \varphi_{G_{\bar{a} \bar{b}}, N}\left(\mathbf{R R}^{\prime} ; j, j^{\prime} ; t, t^{\prime}\right) \beta b\left(j, j^{\prime} ; t^{\prime}\right) \mathrm{d} t^{\prime} \\
& +\sum_{b^{\prime}} \int \mathrm{d} \mathbf{r}^{\prime} \int_{-\infty}^{t} \mathrm{e}^{\epsilon\left(t^{\prime}-t\right)} \varphi_{G_{\bar{a} \bar{b}} n_{b^{\prime}}}^{\nu \mu}\left(\mathbf{R} \mathbf{R}^{\prime}, \mathbf{r}^{\prime} ; t, t^{\prime}\right) \beta \mu_{b^{\prime}}\left(\mathbf{r}^{\prime} ; t^{\prime}\right) \mathrm{d} t^{\prime} \\
& +\sum_{\bar{b}^{\prime}} \sum_{\nu^{\prime}} \int \mathrm{d} \mathbf{R}^{\prime \prime} \int_{-\infty}^{t} \mathrm{e}^{\epsilon\left(t^{\prime}-t\right)} \varphi_{G_{\bar{a} \bar{b}} \bar{b}_{\bar{b}^{\prime}}}^{\nu \mu \nu^{\prime}}\left(\mathbf{R} \mathbf{R}^{\prime}, \mathbf{R}^{\prime \prime} ; t, t^{\prime}\right) \beta \mu_{\bar{b}^{\prime}}^{\nu^{\prime}}\left(\mathbf{R}^{\prime} ; t^{\prime}\right) \mathrm{d} t^{\prime} \\
& +\sum_{b^{\prime}} \int \mathrm{d} \mathbf{r}^{\prime} \int_{-\infty}^{t} \mathrm{e}^{\epsilon\left(t^{\prime}-t\right)} \varphi_{G_{\bar{a} \bar{b}} d_{b^{\prime}}}\left(\mathbf{R} \mathbf{R}^{\prime}, \mathbf{r}^{\prime} ; t, t^{\prime}\right) \beta \mathbf{e}\left(\mathbf{r}^{\prime} ; t^{\prime}\right) \mathrm{d} t^{\prime} \\
& +\sum_{\bar{a}^{\prime} \bar{b}^{\prime}} \sum_{\nu^{\prime} \mu^{\prime}} \int \mathrm{d} \mathbf{R}^{\prime \prime} \int \mathrm{d} \mathbf{R}^{\prime \prime \prime} \\
& \times \int_{-\infty}^{t} \mathrm{e}^{\epsilon\left(t^{\prime}-t\right)} \varphi_{G_{\bar{a} \bar{b}} \nu_{\bar{a}^{\prime} \bar{b}^{\prime}}}^{\nu \nu^{\prime} \mu^{\prime}}\left(\mathbf{R R}^{\prime}, \mathbf{R}^{\prime \prime} \mathbf{R}^{\prime \prime \prime} ; t, t^{\prime}\right) \beta M_{\bar{a}^{\prime} \bar{b}^{\prime}}^{\nu^{\prime} \mu^{\prime}}\left(\mathbf{R}^{\prime \prime} \mathbf{R}^{\prime \prime \prime} ; t^{\prime}\right) \mathrm{d} t^{\prime}
\end{aligned}
$$

where $\varphi_{N N}, \varphi_{n_{a} n_{b}}, \varphi_{n_{\bar{a}} n_{\bar{a}}}^{\nu \nu^{\prime}}, \varphi_{N n_{a}}, \varphi_{N n_{\bar{a}}}^{\nu^{\prime}}, \varphi_{n_{a} n_{\bar{a}}}^{\nu^{\prime}}, \varphi_{d_{a} d_{b}}, \varphi_{G_{\bar{a} \bar{b}} G_{\bar{a}}{ }^{\prime} \bar{b}}^{\nu \mu \nu^{\prime} \prime^{\prime}}$ are generalized transport cores which describe dissipative processes in the system. Transport cores are built in the generalized flows (2.15) and have the following structure:

$$
\varphi_{B B^{\prime}}\left(t, t^{\prime}\right)=\operatorname{Sp}\left(I_{B}(t) T\left(t, t^{\prime}\right) \int_{0}^{1} \mathrm{~d} \tau \rho_{q}^{\tau}\left(t^{\prime}\right) I_{B^{\prime}}\left(t^{\prime}\right) \rho_{q}^{1-\tau}\left(t^{\prime}\right)\right)
$$

In particular, the transport core $\varphi_{N N}\left(l l^{\prime}, j j^{\prime} ; t, t^{\prime}\right)$ describes dynamical dissipative interelectron flow correlations, $\varphi_{n_{a} n_{b}}\left(\mathbf{r}, \mathbf{r}^{\prime} ; t, t^{\prime}\right)$ describes dynamical correlations of 
diffusion flows of gas atoms and, as it will be shown below, is connected with the nonuniform diffusion coefficient $D_{a b}\left(\mathbf{r}, \mathbf{r}^{\prime} ; t\right)$ of gas atoms or molecules. Similarly, the transport core $\varphi_{n_{\bar{a}} n_{\bar{b}}}^{\nu \nu^{\prime}}\left(\mathbf{R}, \mathbf{R}^{\prime} ; t, t^{\prime}\right)$ describes dynamical dissipative correlations of diffusion flows of gas atoms in states $\nu$ and $\nu^{\prime}$ on a metal surface and defines nonuniform diffusion coefficients $D_{\bar{a} \bar{b}}^{\nu \nu^{\prime}}\left(\mathbf{R}, \mathbf{R}^{\prime} ; t\right)$ of adsorbed atoms on a metal surface. Another transport core describes dynamical dissipative correlations between generalized electron flow $I_{N}\left(l, l^{\prime} ; t\right)$, the flow of gas atoms $I_{n_{a}}(\mathbf{r} ; t)$ and of adsorbed atoms $I_{n_{\mathbf{a}}}^{\nu}(\mathbf{R} ; t)$. In particular, transport cores $\varphi_{n_{\bar{a}} n_{b}}^{\nu}\left(\mathbf{R} ; \mathbf{r}^{\prime} ; t, t^{\prime}\right), \varphi_{n_{a} n_{\bar{b}}}^{\nu^{\prime}}\left(\mathbf{r} ; \mathbf{R} ; t, t^{\prime}\right)$ describe dissipative correlations between the flows of gas atoms and adsorbed atoms and define nonuniform coefficients of mutual diffusion $D_{a \bar{b}}^{\nu^{\prime}}\left(\mathbf{r}, \mathbf{R}^{\prime} ; t\right)$ "gas atom - adsorbed atom". The study of these diffusion coefficients in adsorption processes is very important.

The $\varphi_{d_{a} d_{b}}\left(\mathbf{r}, \mathbf{r}^{\prime} ; t, t^{\prime}\right)$ are responsible for the polarization effects in molecules which are induced by a dynamic electric field of the metal surface. The transport cores $\varphi_{G_{\bar{a} \bar{b} p}}^{\nu \mu}\left(\mathbf{R R}^{\prime} ; t, t^{\prime}\right)\{p=N, n, \bar{n}, d\}$ describe dissipative correlations between the adsorbed atoms densities and flow densities of electrons on the metal surface, atoms and molecules of the gas, adsorbed atoms as well as the molecules polarization. $\varphi_{G_{\bar{a} \bar{b}} G_{\bar{a}}^{\prime} \bar{b}}^{\nu \nu \nu^{\prime} \mu^{\prime}}\left(\mathbf{R R}^{\prime}, \mathbf{R}^{\prime \prime} \mathbf{R}^{\prime \prime \prime} ; t, t^{\prime}\right)$ describe diffusion-reaction processes between the adsorbed atoms on the metal surface. They are higher memory functions with respect to dynamical variables $G_{\bar{a} \bar{b}}^{\nu \mu}$. The calculation of these transport cores appears to be a well-known problem of nonequilibrium statistical mechanics. Thus, we obtained generalized transport equations (2.16)-(2.20) for a one-electron density matrix, average nonequilibrium densities of adsorbed and non-adsorbed gas atoms for the consistent study of kinetic electron and atomic diffusion-reaction processes in a system "metal-adsorbate-gas". It can be seen that these equations have a nonlinear and a nonuniform structure, they can describe both strongly and weakly nonequilibrium processes. In further considerations, our primary interest is devoted to the weakly nonequilibrium case.

\section{Weakly nonequilibrium processes}

In such a case, one should suppose that the one-electron density matrix $\left\langle\hat{N}_{l l^{\prime}}\right\rangle^{t}$, average nonequilibrium densities of adsorbed and non-adsorbed gas atoms $\left\langle\hat{n}_{\bar{a}}^{\nu}(\mathbf{R})\right\rangle^{t}$, $\left\langle\hat{n}_{a}(\mathbf{r})\right\rangle^{t}$ and $\left\langle\hat{\mathbf{d}}_{a}(\mathbf{r})\right\rangle^{t},\left\langle\hat{G}_{\bar{a} \bar{b}}^{\nu, \mu}\left(\mathbf{R}, \mathbf{R}^{\prime}\right)\right\rangle^{t}$ correspondingly, reciprocal thermodynamic parameters $b\left(l, l^{\prime} ; t\right), \mu_{\bar{a}}^{\nu}(\mathbf{R} ; t), \mu_{a}(\mathbf{r} ; t), \mathbf{e}(\mathbf{r} ; t), M_{\bar{a} \bar{b}}^{\nu \mu}\left(\mathbf{R}, \mathbf{R}^{\prime} ; t\right)$ deviate slightly from their equilibrium values. Then one can expand the relevant statistical operator (2.11) on the deviations of parameters $b\left(l, l^{\prime} ; t\right), \mu_{\bar{a}}^{\nu}(\mathbf{R} ; t), \mu_{a}(\mathbf{r} ; t), \mathbf{e}(\mathbf{r} ; t), M_{\bar{a} \bar{b}}^{\nu \mu}\left(\mathbf{R}, \mathbf{R}^{\prime} ; t\right)$ from their equilibrium values $b_{0}\left(l, l^{\prime}\right), \mu_{\bar{a}}^{\nu}(\mathbf{R}), \mu_{a}(\mathbf{r}), M_{\bar{a} \bar{b}}^{\nu \mu}\left(\mathbf{R}, \mathbf{R}^{\prime}\right)$ and restrict the expansion to the line of approximation. Then from (2.16)-(2.20) one obtains a transport equation in the linear approximation, using the Laplace transformation for time $t>0$,

$$
\langle\hat{A}\rangle_{z}=\mathrm{i} \int_{0}^{\infty} \mathrm{e}^{\mathrm{i} z t} \hat{A}(t) \mathrm{d} t, \quad z=\omega+\mathrm{i} \varepsilon
$$




$$
\begin{aligned}
& z\left\langle\delta \hat{p}_{n}\right\rangle_{z}-\sum_{j, j^{\prime}} \Omega_{p_{n} N}\left(j, j^{\prime} ; z\right)\left\langle\delta \hat{N}_{j, j^{\prime}}\right\rangle_{z}-\sum_{b} \int \mathrm{d} \mathbf{r}^{\prime} \Omega_{p_{n} n_{b}}\left(\mathbf{r}^{\prime} ; z\right)\left\langle\delta \bar{n}_{b}\left(\mathbf{r}^{\prime}\right)\right\rangle_{z} \\
& -\sum_{\bar{b}} \sum_{\nu^{\prime}} \int \mathrm{d} \mathbf{R}^{\prime} \Omega_{p_{n} n_{\bar{b}}}^{\nu^{\prime}}\left(\mathbf{R}^{\prime} ; z\right)\left\langle\delta \bar{n}_{\bar{b}}^{\nu^{\prime}}\left(\mathbf{R}^{\prime}\right)\right\rangle_{z}-\sum_{b} \int \mathrm{d} \mathbf{r}^{\prime} \Omega_{p_{n} d_{b}}\left(\mathbf{r}^{\prime} ; z\right)\left\langle\delta \overline{\mathbf{d}}_{b}\left(\mathbf{r}^{\prime}\right)\right\rangle_{z}
\end{aligned}
$$

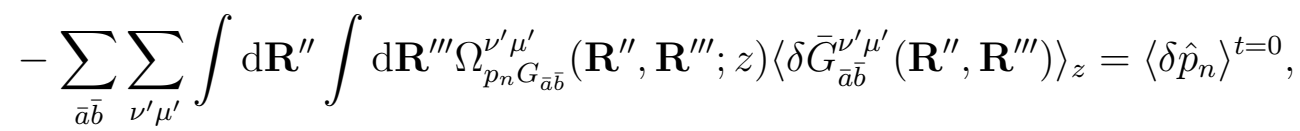

where $\delta \hat{p}_{n}=\left(\delta \hat{N}_{l, l^{\prime}}, \delta \bar{n}_{a}(\mathbf{r}), \delta \bar{n}_{\bar{a}}^{\nu}(\mathbf{R}), \delta \overline{\mathbf{d}}_{a}(\mathbf{r}), \delta \bar{G}_{\bar{a} \bar{b}}^{\nu \mu}\left(\mathbf{R}, \mathbf{R}^{\prime}\right)\right)$. Here

$$
\begin{aligned}
& \delta \hat{N}_{l l^{\prime}}=\hat{N}_{l l^{\prime}}-\left\langle\hat{N}_{l l^{\prime}}\right\rangle_{0}, \quad \delta \bar{n}_{a}(\mathbf{r})=\bar{n}_{a}(\mathbf{r})-\left\langle\bar{n}_{a}(\mathbf{r})\right\rangle_{0}, \quad \delta \bar{n}_{\bar{a}}^{\nu}(\mathbf{R})=\bar{n}_{\bar{a}}^{\nu}(\mathbf{R})-\left\langle\bar{n}_{\bar{a}}^{\nu}(\mathbf{R})\right\rangle_{0}, \\
& \delta \overline{\mathbf{d}}_{a}(\mathbf{r})=\overline{\mathbf{d}}_{a}(\mathbf{r})-\left\langle\overline{\mathbf{d}}_{a}(\mathbf{r})\right\rangle_{0}, \quad \delta \bar{G}_{\bar{a} \bar{b}}^{\nu \mu}\left(\mathbf{R}, \mathbf{R}^{\prime}\right)=\bar{G}_{\bar{a} \bar{b}}^{\nu \mu}\left(\mathbf{R}, \mathbf{R}^{\prime}\right)-\left\langle\bar{G}_{\bar{a} \bar{b}}^{\nu \mu}\left(\mathbf{R}, \mathbf{R}^{\prime}\right)\right\rangle_{0},
\end{aligned}
$$

where average values are calculated with the use of equilibrium statistical operator

$$
\begin{aligned}
& \rho_{0}=Z^{-1} \exp \left\{-\beta\left(H-\sum_{l l^{\prime}} b_{0}\left(l, l^{\prime}\right) \hat{N}_{l l^{\prime}}-\sum_{a} \int \mathrm{d} \mathbf{r} \mu_{a}(\mathbf{r}) \hat{n}_{a}(\mathbf{r})\right.\right. \\
& \left.\left.-\sum_{\bar{a}} \sum_{\nu} \int \mathrm{d} \mathbf{R} \mu_{\bar{a}}^{\nu}(\mathbf{R}) \hat{n}_{\bar{a}}^{\nu}(\mathbf{R})-\sum_{\bar{a} \bar{b}} \sum_{\nu \mu} \int \mathrm{d} \mathbf{R} \mathrm{d} \mathbf{R}^{\prime} M_{\bar{a} \bar{b}}^{\nu, \mu}\left(\mathbf{R}, \mathbf{R}^{\prime}\right) \hat{G}_{\bar{a} \bar{b}}^{\nu, \mu}\left(\mathbf{R}, \mathbf{R}^{\prime}\right)\right)\right\} \\
& Z=\operatorname{Sp} \exp \left\{-\beta\left(H-\sum_{l l^{\prime}} b_{0}\left(l, l^{\prime}\right) \hat{N}_{l l^{\prime}}-\sum_{a} \int \mathrm{d} \mathbf{r} \mu_{a}(\mathbf{r}) \hat{n}_{a}(\mathbf{r})\right.\right. \\
& \left.\left.-\sum_{a} \sum_{\nu} \int \mathrm{d} \mathbf{R} \mu_{\bar{a}}^{\nu}(\mathbf{R}) \hat{n}_{\bar{a}}^{\nu}(\mathbf{R})\right)-\sum_{\bar{a} \bar{b}} \sum_{\nu \mu} \int \mathrm{d} \mathbf{R} d \mathbf{R}^{\prime} M_{\bar{a} \bar{b}}^{\nu, \mu}\left(\mathbf{R}, \mathbf{R}^{\prime}\right) \hat{G}_{\bar{a} \bar{b}}^{\nu, \mu}\left(\mathbf{R}, \mathbf{R}^{\prime}\right)\right\}
\end{aligned}
$$

is the grand partition sum of the system "metal-adsorbate-gas". $\mu_{a}(\mathbf{r}), \mu_{\bar{a}}^{\nu}(\mathbf{R})$ are local equilibrium values of the chemical potentials of non-adsorbed and adsorbed gas atoms, $\langle\ldots\rangle_{0}=\operatorname{Sp}\left(\ldots \rho_{0}\right)$. Exclusion of parameters $\beta \delta b\left(l, l^{\prime} ; t\right), \beta \delta \mu_{a}(\mathbf{r} ; t), \beta \delta \mu_{\bar{a}}^{\nu}(\mathbf{R} ; t)$ and $M_{\bar{a} \bar{b}}^{\nu, \mu}\left(\mathbf{R}, \mathbf{R}^{\prime}\right)$ in $\rho_{q}(t)^{\prime}$ using of self-consistency conditions (2.12) results in the appearing of corresponding orthogonal variables

$$
\begin{aligned}
\bar{n}_{a}(\mathbf{r}) & =\hat{n}_{a}(\mathbf{r})-\sum_{\substack{l, l^{\prime} \\
j, j^{\prime}}}\left\langle\hat{n}_{a}(\mathbf{r}) \hat{N}_{l l^{\prime}}\right\rangle_{0} \Phi_{N N}^{-1}\left(l, l^{\prime} ; j, j^{\prime}\right) \hat{N}_{j j^{\prime}} \\
\bar{n}_{\bar{a}}^{\nu}(\mathbf{R}) & =\hat{n}_{\bar{a}}^{\nu}(\mathbf{R})-\sum_{\substack{l, l^{\prime} \\
j, j^{\prime}}}\left\langle\hat{n}_{\bar{a}}^{\nu}(\mathbf{R}) \hat{N}_{l l^{\prime}}\right\rangle_{0} \Phi_{N N}^{-1}\left(l, l^{\prime} ; j, j^{\prime}\right) \hat{N}_{j j^{\prime}} \\
& -\sum_{a b} \int \mathrm{d} \mathbf{r} \int \mathrm{d} \mathbf{r}^{\prime}\left\langle\hat{n}_{\bar{a}}^{\nu}(\mathbf{R}) \bar{n}_{a}(\mathbf{r})\right\rangle_{0}\left[\Phi_{n n}^{-1}\left(\mathbf{r r}^{\prime}\right)\right]_{a b} \bar{n}_{b}\left(\mathbf{r}^{\prime}\right) \\
\overline{\mathbf{d}}_{a}(\mathbf{r}) & =\hat{\mathbf{d}}_{a}(\mathbf{r})-\sum_{\substack{l, l^{\prime} \\
j, j^{\prime}}}\left\langle\hat{\mathbf{d}}_{a}(\mathbf{r}) \hat{N}_{l l^{\prime}}\right\rangle_{0} \Phi_{N N}^{-1}\left(l, l^{\prime} ; j, j^{\prime}\right) \hat{N}_{j j^{\prime}} \\
& -\sum_{a^{\prime} b} \int \mathrm{d} \mathbf{r}^{\prime} \int \mathrm{d} \mathbf{r}^{\prime \prime}\left\langle\hat{\mathbf{d}}_{a}(\mathbf{r}) \bar{n}_{a^{\prime}}\left(\mathbf{r}^{\prime}\right)\right\rangle_{0}\left[\Phi_{n n}^{-1}\left(\mathbf{r}^{\prime} \mathbf{r}^{\prime \prime}\right)\right]_{a^{\prime} b} \bar{n}_{b}\left(\mathbf{r}^{\prime \prime}\right)
\end{aligned}
$$




$$
\begin{aligned}
&\left.-\sum_{\bar{a} \bar{b}} \sum_{\nu^{\prime} \mu^{\prime}} \int \mathrm{d} \mathbf{R}^{\prime} \int \mathrm{d} \mathbf{R}^{\prime \prime}\left\langle\hat{\mathbf{d}}_{a}(\mathbf{r}) \bar{n}_{\bar{a}}^{\nu^{\prime}} \mathbf{R}^{\prime}\right)\right\rangle_{0}\left[\Phi_{n n}^{-1}\left(\mathbf{R}^{\prime} \mathbf{R}^{\prime \prime}\right)\right]_{\bar{a} \bar{b}}^{\nu^{\prime} \mu^{\prime}} \bar{n}_{\bar{b}}^{\mu^{\prime}}\left(\mathbf{R}^{\prime \prime}\right), \\
& \bar{G}_{\bar{a} \bar{b}}^{\nu \mu}\left(\mathbf{R}, \mathbf{R}^{\prime}\right)=\hat{G}_{\bar{a} \bar{b}}^{\nu \mu}\left(\mathbf{R}, \mathbf{R}^{\prime}\right)-\sum_{\substack{l, l^{\prime} \\
j, j^{\prime}}}\left\langle\hat{G}_{\bar{a} \bar{b}}^{\nu \mu}\left(\mathbf{R}, \mathbf{R}^{\prime}\right) \hat{N}_{l l^{\prime}}\right\rangle_{0} \Phi_{N N}^{-1}\left(l, l^{\prime} ; j, j^{\prime}\right) \hat{N}_{j j^{\prime}} \\
& \quad-\sum_{a^{\prime} b} \int \mathrm{d} \mathbf{r}^{\prime} \int \mathrm{d} \mathbf{r}^{\prime \prime}\left\langle\hat{G}_{\bar{a} \bar{b}}^{\nu \mu}\left(\mathbf{R}, \mathbf{R}^{\prime}\right) \bar{n}_{a^{\prime}}\left(\mathbf{r}^{\prime}\right)\right\rangle_{0}\left[\Phi_{n n}^{-1}\left(\mathbf{r}^{\prime} \mathbf{r}^{\prime \prime}\right)\right]_{a^{\prime} b} \bar{n}_{b}\left(\mathbf{r}^{\prime \prime}\right) \\
&\left.-\sum_{\bar{a}^{\prime} \bar{b}^{\prime}} \sum_{\nu^{\prime} \mu^{\prime}} \int \mathrm{d} \mathbf{R}^{\prime \prime} \int \mathrm{d} \mathbf{R}^{\prime \prime \prime}\left\langle\hat{G}_{\bar{a} \bar{b}}^{\nu \mu}\left(\mathbf{R}, \mathbf{R}^{\prime}\right) \bar{n}_{\bar{a}^{\prime}}^{\nu^{\prime}} \mathbf{R}^{\prime \prime}\right)\right\rangle_{0}\left[\Phi_{n n}^{-1}\left(\mathbf{R}^{\prime \prime} \mathbf{R}^{\prime \prime \prime}\right)\right]_{\bar{a}^{\prime} \bar{b}^{\prime}}^{\nu^{\prime} \mu^{\prime}} \bar{n}_{b}^{\mu^{\prime}}\left(\mathbf{R}^{\prime \prime \prime}\right) \\
&-\sum_{a^{\prime} b} \int \mathrm{d} \mathbf{r}^{\prime} \int \mathrm{d} \mathbf{r}^{\prime \prime}\left\langle\hat{G}_{\bar{a} \bar{b}}^{\nu \mu}\left(\mathbf{R}, \mathbf{R}^{\prime}\right) \overline{\mathbf{d}}_{a^{\prime}}\left(\mathbf{r}^{\prime}\right)\right\rangle_{0}\left[\Phi_{d d}^{-1}\left(\mathbf{r}^{\prime} \mathbf{r}^{\prime \prime}\right)\right]_{a^{\prime} b} \overline{\mathbf{d}}_{b}\left(\mathbf{r}^{\prime \prime}\right) .
\end{aligned}
$$

Conditions of orthogonality for these variables are valid:

$$
\begin{aligned}
& \left\langle\bar{n}_{a}(\mathbf{r}) \hat{N}_{j j^{\prime}}\right\rangle_{0}=0, \quad\left\langle\bar{n}_{\bar{a}}^{\nu}(\mathbf{R}) \hat{N}_{j j^{\prime}}\right\rangle_{0}=0, \\
& \left\langle\bar{n}_{a}(\mathbf{r}) \bar{n}_{\bar{a}}^{\nu}(\mathbf{R})\right\rangle_{0}=0, \quad\left\langle\bar{n}_{a}(\mathbf{r}) \overline{\mathbf{d}}_{a^{\prime}}\left(\mathbf{r}^{\prime}\right)\right\rangle_{0}=0, \quad\left\langle\bar{n}_{a}(\mathbf{r}) \bar{G}_{\bar{a} \bar{b}}^{\nu \mu}\left(\mathbf{R}, \mathbf{R}^{\prime}\right)\right\rangle_{0}=0 .
\end{aligned}
$$

The functions $\Phi_{N N}^{-1}\left(l, l^{\prime} ; j, j^{\prime}\right),\left[\Phi_{n n}^{-1}\left(\mathbf{r}, \mathbf{r}^{\prime}\right)\right]_{a b}$ and $\left[\Phi_{n n}^{-1}\left(\mathbf{R}, \mathbf{R}^{\prime}\right)\right]_{\bar{a} \bar{b}}^{\nu \mu}$ are inverse to corresponding equilibrium correlation functions:

$$
\Phi\left(l l^{\prime}, j j^{\prime}\right)=\left\langle\hat{N}_{l l^{\prime}} \hat{N}_{j j^{\prime}}\right\rangle_{0},
$$

for the electron and gas subsystems

$$
\begin{aligned}
\Phi_{a b}\left(\mathbf{r}, \mathbf{r}^{\prime}\right) & =\left\langle\bar{n}_{a}(\mathbf{r}) \bar{n}_{b}\left(\mathbf{r}^{\prime}\right)\right\rangle_{0}, \\
\Phi_{\bar{a} \bar{b}}^{\nu \mu}\left(\mathbf{R}, \mathbf{R}^{\prime}\right) & =\left\langle\bar{n}_{\bar{a}}^{\nu}(\mathbf{R}) \bar{n}_{\bar{b}}^{\mu}\left(\mathbf{R}^{\prime}\right)\right\rangle_{0}
\end{aligned}
$$

and are defined from corresponding integral relations [19].

$$
\Omega_{A B}(z)=\mathrm{i} \Omega_{A B}-\varphi_{A B}(z)
$$

i $\Omega_{A B}$ are normalized static correlation functions, they read:

$$
\mathrm{i} \Omega_{A B}=\langle\dot{\hat{A}} \hat{B}\rangle_{0} \Phi_{B B}^{-1},
$$

where $\hat{B}, \hat{A}=\left\{\hat{N}_{l l^{\prime}}, \bar{n}_{a}(\mathbf{r}), \bar{n}_{\bar{a}}(\mathbf{R})\right\}, \overline{\mathbf{d}}_{a^{\prime}}\left(\mathbf{r}^{\prime}\right), \bar{G}_{\bar{a} \bar{b}}^{\nu \mu}\left(\mathbf{R}, \mathbf{R}^{\prime}\right), \dot{\hat{A}}=\mathrm{i} L_{N} \hat{A} . \varphi_{A B}\left(t, t^{\prime}\right)$ are normalized transport cores with the following structure:

$$
\begin{aligned}
\bar{\varphi}_{A B}\left(t, t^{\prime}\right) & =\left\langle\bar{I}_{A} T_{0}\left(t, t^{\prime}\right) \bar{I}_{B}\right\rangle_{0} \Phi_{A B}^{-1} \\
\bar{I}_{A}, \bar{I}_{B} & =\left\{\bar{I}_{N}\left(l, l^{\prime}\right), \bar{I}_{a}(\mathbf{r}), \bar{I}_{\bar{a}}^{\nu}(\mathbf{R}) \bar{I}_{d}^{a}(\mathbf{r}), \bar{I}_{\left.G_{\bar{a} \bar{b}}^{\nu \mu}\left(\mathbf{R R}^{\prime}\right)\right\}}\right.
\end{aligned}
$$

where

$$
T_{0}\left(t, t^{\prime}\right)=\exp \left\{-\left(t^{\prime}-t\right)\left(1-\mathcal{P}_{0}\right) \mathrm{i} L_{N}\right\}
$$


is the evolution operator in the linear approximation;

$$
\begin{aligned}
& \bar{I}_{N}\left(l, l^{\prime}\right)=\left(1-\mathcal{P}_{0}\right) \dot{\hat{N}}_{l l^{\prime}}, \\
& \bar{I}_{a}(\mathbf{r})=\left(1-\mathcal{P}_{0}\right) \dot{\bar{n}}_{a}(\mathbf{r}), \\
& \bar{I}_{\bar{a}}^{\nu}(\mathbf{R})=\left(1-\mathcal{P}_{0}\right) \dot{\bar{n}}_{\bar{a}}^{\nu}(\mathbf{R}) \\
& \bar{I}_{d}^{a}(\mathbf{r})=\left(1-\mathcal{P}_{0}\right) \dot{\overline{\mathbf{d}}}_{a^{\prime}}\left(\mathbf{r}^{\prime}\right), \\
& \bar{I}_{G_{\bar{a} \bar{b}}}^{\nu \mu}\left(\mathbf{R R}^{\prime}\right)=\left(1-\mathcal{P}_{0}\right) \dot{\bar{G}}_{\bar{a} \bar{b}}^{\nu \mu}\left(\mathbf{R}, \mathbf{R}^{\prime}\right)
\end{aligned}
$$

are generalized flows in the linear approximation, where the Mori projection operator $\mathcal{P}_{0}$ has the following structure

$$
\begin{aligned}
& \mathcal{P}_{0}(\ldots)=\langle\ldots\rangle_{0}+\sum_{\substack{l, l^{\prime} \\
j, j^{\prime}}}\left\langle\ldots \hat{N}_{l l^{\prime}}\right\rangle_{0} \Phi^{-1}\left(l, l^{\prime} ; j, j^{\prime}\right) \hat{N}_{j j^{\prime}} \\
& +\sum_{a b} \int \mathrm{d} \mathbf{r} \int \mathrm{d} \mathbf{r}^{\prime}\left\langle\ldots \bar{n}_{a}(\mathbf{r})\right\rangle_{0}\left[\Phi_{n n}^{-1}\left(\mathbf{r}, \mathbf{r}^{\prime}\right)\right]_{a b} \bar{n}_{b}\left(\mathbf{r}^{\prime}\right) \\
& +\sum_{\bar{a} \bar{b}} \sum_{\nu \nu^{\prime}} \int \mathrm{d} \mathbf{R} \int \mathrm{d} \mathbf{R}^{\prime}\left\langle\ldots \bar{n}_{\bar{a}}^{\nu}(\mathbf{R})\right\rangle_{0}\left[\Phi_{n n}^{-1}\left(\mathbf{R}, \mathbf{R}^{\prime}\right)\right]_{\bar{a} \bar{b}}^{\nu \nu^{\prime}} \bar{n}_{\bar{b}}^{\nu^{\prime}}\left(\mathbf{R}^{\prime}\right) \\
& +\sum_{a^{\prime} b} \int \mathrm{d} \mathbf{r}^{\prime} \int \mathrm{d} \mathbf{r}^{\prime \prime}\left\langle\ldots \overline{\mathbf{d}}_{a^{\prime}}\left(\mathbf{r}^{\prime}\right)\right\rangle_{0}\left[\Phi_{d d}^{-1}\left(\mathbf{r}^{\prime} \mathbf{r}^{\prime \prime}\right)\right]_{a^{\prime} b} \overline{\mathbf{d}}_{b}\left(\mathbf{r}^{\prime \prime}\right) \\
& +\sum_{\bar{a} \bar{b}} \sum_{\bar{a}^{\prime} \bar{b}^{\prime}} \sum_{\nu \nu^{\prime}} \sum_{\mu \mu^{\prime}} \int \mathrm{d} \mathbf{R} \int \mathrm{d} \mathbf{R}^{\prime} \int \mathrm{d} \mathbf{R}^{\prime \prime} \int \mathrm{d} \mathbf{R}^{\prime \prime \prime}\left\langle\ldots \bar{G}_{\bar{a} \bar{b}}^{\nu \nu^{\prime}}\left(\mathbf{R}, \mathbf{R}^{\prime}\right)\right\rangle_{0} \\
& \times\left[\Phi_{G G}\left(\mathbf{R}, \mathbf{R}^{\prime}, \mathbf{R}^{\prime \prime}, \mathbf{R}^{\prime \prime \prime}\right)\right]_{\bar{a} \overline{\bar{a}} \bar{a}^{\prime} \bar{b}^{\prime}}^{\nu \mu^{\prime}} \bar{G}_{\bar{a}^{\prime} \bar{b}^{\prime}}^{\mu \mu^{\prime}}\left(\mathbf{R}^{\prime \prime}, \mathbf{R}^{\prime \prime \prime}\right) .
\end{aligned}
$$

Its operator properties are as usual:

$$
\begin{aligned}
& \mathcal{P}_{0} \mathcal{P}_{0}=\mathcal{P}_{0}, \quad \mathcal{P}_{0}\left(1-\mathcal{P}_{0}\right)=0 \\
& \mathcal{P}_{0} \hat{N}_{l l^{\prime}}=\hat{N}_{l l^{\prime}}, \quad \mathcal{P}_{0} \bar{n}_{a}(\mathbf{r})=\bar{n}_{a}(\mathbf{r}), \\
& \mathcal{P}_{0} \bar{n}_{\bar{a}}^{\nu}(\mathbf{R})=\bar{n}_{\bar{a}}^{\nu}(\mathbf{R}), \quad \mathcal{P}_{0} \overline{\mathbf{d}}_{a}(\mathbf{r})=\overline{\mathbf{d}}_{a}(\mathbf{r}), \quad \mathcal{P}_{0} \bar{G}_{\bar{a} \bar{b}}^{\nu \nu^{\prime}}\left(\mathbf{R}, \mathbf{R}^{\prime}\right)=\bar{G}_{\bar{a}}^{\nu \nu^{\prime}}\left(\mathbf{R}, \mathbf{R}^{\prime}\right) .
\end{aligned}
$$

The set of transport equations for weakly nonequilibrium case is linear, closed and describes kinetic electron and atomic diffusion-reaction processes consistently. The functions $i \Omega_{A B}(2.22)$ are static correlation functions and can be expressed via the corresponding interparticle potentials of interactions and structural equilibrium distribution functions of electrons and atoms of our system. $\bar{\varphi}_{A B}\left(t, t^{\prime}\right)$ are time correlation functions which are built on generalized flows and describe dissipative processes in a system. In particular, $\bar{\varphi}_{N N}\left(l, l^{\prime} ; j, j^{\prime} ; t, t^{\prime}\right)$ describe interelectron dissipative processes, $\bar{\varphi}_{a a}\left(\mathbf{r}, \mathbf{r}^{\prime} ; t, t^{\prime}\right), \bar{\varphi}_{\bar{a} \bar{a}}^{\nu \nu^{\prime}}\left(\mathbf{R}, \mathbf{R}^{\prime} ; t, t^{\prime}\right)$ describe nonuniform diffusion processes of adsorbed and non-adsorbed gas atoms. All other memory functions describe cross dissipative correlations of flows of electrons and atoms in the spatially inhomogeneous system "metal-adsorbate-gas". The set of transport equations (2.22) permits limiting cases. In particular, if formally one neglects the diffusion-reaction processes 
of adsorbed and non-adsorbed gas atoms, then electron kinetics in a system of metal surface is described by an equation for the nonequilibrium one-electron density matrix. Using the properties $\delta \hat{p}_{n}=\delta \hat{N}_{l, l^{\prime}}$ in the equation (2.22), such an equation can be represented in a form

$$
z\left\langle\delta \hat{N}_{l l^{\prime}}\right\rangle_{z}-\sum_{j, j^{\prime}} \Omega_{N N}\left(l, l^{\prime} ; j, j^{\prime} ; z\right)\left\langle\delta \hat{N}_{j, j^{\prime}}\right\rangle_{z}=\left\langle\delta \hat{N}_{l l^{\prime}}\right\rangle^{t=0}
$$

where

$$
\Omega_{N N}\left(l, l^{\prime} ; j, j^{\prime} ; z\right)=\mathrm{i} \Omega_{N N}\left(l, l^{\prime} ; j, j^{\prime}\right)-\bar{\varphi}_{N N}\left(l, l^{\prime} ; j, j^{\prime} ; z\right)
$$

is the mass operator of an electron subsystem. This set of equations permits to define elements of one-electron density matrix $\left\langle\hat{a}_{f \nu \sigma}^{+} \hat{p}_{l \xi \sigma^{\prime}}\right\rangle^{t}$. Electron tunnelling current between positions $f$ and $l$ on a metal surface is then expressed via these elements. Another limiting case can be obtained if one formally does not take into consideration the electron kinetic processes, whereas the interaction of the adsorbed atoms and the substrate is considered to be classical only. Then the set (2.22) transfers to a set of nonuniform diffusion-reaction equations of adsorbed and non-adsorbed gas atoms:

$$
\begin{aligned}
& \frac{\partial}{\partial t}\left\langle\delta \hat{n}_{a}(\mathbf{r})\right\rangle^{t}=-\sum_{b} \int \mathrm{d} \mathbf{r}^{\prime} \int_{-\infty}^{t} \mathrm{e}^{\epsilon\left(t^{\prime}-t\right)} \frac{\partial}{\partial \mathbf{r}} D_{a b}\left(\mathbf{r}, \mathbf{r}^{\prime} ; t, t^{\prime}\right) \frac{\partial}{\partial \mathbf{r}^{\prime}}\left\langle\delta \hat{n}_{b}\left(\mathbf{r}^{\prime}\right)\right\rangle^{t^{\prime}} \mathrm{d} t^{\prime} \\
& +\sum_{\bar{b}} \sum_{\nu^{\prime}} \int \mathrm{d} \mathbf{R}^{\prime} \int_{-\infty}^{t} \mathrm{e}^{\epsilon\left(t^{\prime}-t\right)} \frac{\partial}{\partial \mathbf{r}} D_{a \bar{b}}^{\nu^{\prime}}\left(\mathbf{r}, \mathbf{R}^{\prime} ; t, t^{\prime}\right) \frac{\partial}{\partial \mathbf{R}^{\prime}}\left\langle\delta \bar{n}_{\bar{b}}^{\nu^{\prime}}\left(\mathbf{R}^{\prime}\right)\right\rangle^{t^{\prime}} \mathrm{d} t^{\prime} \\
& +\sum_{\bar{a} \bar{b}} \sum_{\nu^{\prime} \mu^{\prime}} \int \mathrm{d} \mathbf{R}^{\prime \prime} \int \mathrm{d} \mathbf{R}^{\prime \prime \prime} \\
& \times \int_{-\infty}^{t} \mathrm{e}^{\epsilon\left(t^{\prime}-t\right)} \frac{\partial}{\partial \mathbf{r}} K_{n_{a} G_{\bar{a} \bar{b}}}^{\nu^{\prime} \mu^{\prime}}\left(\mathbf{r} ; \mathbf{R}^{\prime \prime}, \mathbf{R}^{\prime \prime \prime} ; t, t^{\prime}\right)\left\langle\delta \bar{G}_{\bar{a} \bar{b}}^{\nu^{\prime} \mu^{\prime}}\left(\mathbf{R}^{\prime \prime}, \mathbf{R}^{\prime \prime \prime}\right)\right\rangle^{t^{\prime}} \mathrm{d} t^{\prime} \\
& \frac{\partial}{\partial t}\left\langle\delta \bar{n}_{\bar{a}}^{\nu}(\mathbf{R})\right\rangle^{t}=-\sum_{\bar{b}} \sum_{\nu^{\prime}} \int \mathrm{d} \mathbf{R}^{\prime} \frac{\partial}{\partial \mathbf{R}} D_{\bar{a} \bar{b}}^{\nu \nu^{\prime}}\left(\mathbf{R}, \mathbf{R}^{\prime} ; t, t^{\prime}\right) \frac{\partial}{\partial \mathbf{R}^{\prime}}\left\langle\delta \bar{n}_{\bar{b}}^{\nu^{\prime}}\left(\mathbf{R}^{\prime}\right)\right\rangle^{t^{\prime}} \mathrm{d} t^{\prime} \\
& -\sum_{b} \int \mathrm{d} \mathbf{r}^{\prime} \frac{\partial}{\partial \mathbf{R}} D_{\bar{a} b}^{\nu}\left(\mathbf{R}, \mathbf{r}^{\prime} ; t, t^{\prime}\right) \frac{\partial}{\partial \mathbf{r}^{\prime}}\left\langle\delta \hat{n}_{b}(\mathbf{r})\right\rangle^{t^{\prime}} \mathrm{d} t^{\prime}+ \\
& +\sum_{\bar{a}^{\prime} \bar{b}} \sum_{\nu^{\prime} \mu^{\prime}} \int \mathrm{d} \mathbf{R}^{\prime \prime} \int \mathrm{d} \mathbf{R}^{\prime \prime \prime} \\
& \times \int_{-\infty}^{t} \mathrm{e}^{\epsilon\left(t^{\prime}-t\right)} \frac{\partial}{\partial \mathbf{R}} K_{n_{\bar{a}} G_{\bar{a}} \bar{b}}^{\nu, \nu^{\prime} \mu^{\prime}}\left(\mathbf{R} ; \mathbf{R}^{\prime \prime}, \mathbf{R}^{\prime \prime \prime} ; t, t^{\prime}\right)\left\langle\delta \bar{G}_{\bar{a}^{\prime} \bar{b}}^{\nu^{\prime} \mu^{\prime}}\left(\mathbf{R}^{\prime \prime}, \mathbf{R}^{\prime \prime \prime}\right)\right\rangle^{t^{\prime}} \mathrm{d} t^{\prime} \\
& \frac{\partial}{\partial t}\left\langle\delta \bar{G}_{\bar{a} \bar{b}}^{\nu \mu}\left(\mathbf{R}, \mathbf{R}^{\prime}\right)\right\rangle^{t}=\sum_{b} \int \mathrm{d} \mathbf{r}^{\prime} \int_{-\infty}^{t} \mathrm{e}^{\epsilon\left(t^{\prime}-t\right)} K_{G_{\bar{a} \bar{b}} n_{b}}^{\nu \mu}\left(\mathbf{R}, \mathbf{R}^{\prime} ; \mathbf{r}^{\prime} ; t, t^{\prime}\right) \frac{\partial}{\partial \mathbf{r}^{\prime}}\left\langle\delta \hat{n}_{b}\left(\mathbf{r}^{\prime}\right)\right\rangle^{t^{\prime}} \mathrm{d} t^{\prime}
\end{aligned}
$$




$$
\begin{aligned}
& +\sum_{\bar{b}^{\prime}} \sum_{\nu^{\prime}} \int \mathrm{d} \mathbf{R}^{\prime \prime} \int_{-\infty}^{t} \mathrm{e}^{\epsilon\left(t^{\prime}-t\right)} K_{G_{\bar{a} \bar{b}} n_{\overline{b^{\prime}}}}^{\nu \mu, \nu^{\prime}}\left(\mathbf{R}, \mathbf{R}^{\prime} ; \mathbf{R}^{\prime \prime} ; t, t^{\prime}\right) \frac{\partial}{\partial \mathbf{R}^{\prime}}\left\langle\delta \bar{n}_{\bar{b}^{\prime}}^{\nu^{\prime}}\left(\mathbf{R}^{\prime \prime}\right)\right\rangle^{t^{\prime}} \mathrm{d} t^{\prime} \\
& -\sum_{\bar{a}^{\prime} \bar{b}^{\prime}} \sum_{\nu^{\prime} \mu^{\prime}} \int \mathrm{d} \mathbf{R}^{\prime \prime} \int \mathrm{d} \mathbf{R}^{\prime \prime \prime} \\
& \quad \times \int_{-\infty}^{t} \mathrm{e}^{\epsilon\left(t^{\prime}-t\right)} K_{G_{\bar{a} \bar{b}} G_{\bar{a}^{\prime} \bar{b}^{\prime}}}^{\nu, \nu^{\prime} \mu^{\prime}}\left(\mathbf{R}, \mathbf{R}^{\prime} ; \mathbf{R}^{\prime \prime}, \mathbf{R}^{\prime \prime \prime} ; t, t^{\prime}\right)\left\langle\delta \bar{G}_{\bar{a}^{\prime} \bar{b}^{\prime} \mu^{\prime}}\left(\mathbf{R}^{\prime \prime}, \mathbf{R}^{\prime \prime \prime}\right)\right\rangle^{t^{\prime}} \mathrm{d} t^{\prime},
\end{aligned}
$$

where

$$
\begin{aligned}
& \bar{n}_{\bar{a}}^{\nu}(\mathbf{R})=\hat{n}_{\bar{a}}^{\nu}(\mathbf{R})-\sum_{a b} \int \mathrm{d} \mathbf{r} \mathrm{d} \mathbf{r}^{\prime}\left\langle\hat{n}_{\bar{a}}^{\nu}(\mathbf{R}) \hat{n}_{a}(\mathbf{r})\right\rangle_{0}\left[\Phi_{n n}^{-1}\right]_{a b}\left(\mathbf{r}, \mathbf{r}^{\prime}\right) \hat{n}_{b}\left(\mathbf{r}^{\prime}\right) \\
& \bar{G}_{\bar{a} \bar{b}}^{\nu \mu}\left(\mathbf{R}, \mathbf{R}^{\prime}\right)=\hat{G}_{\bar{a} \bar{b}}^{\nu \mu}\left(\mathbf{R}, \mathbf{R}^{\prime}\right)-\sum_{a^{\prime} b} \int \mathrm{d} \mathbf{r}^{\prime} \int \mathrm{d} \mathbf{r}^{\prime \prime}\left\langle\hat{G}_{\bar{a} \bar{b}}^{\nu \mu}\left(\mathbf{R}, \mathbf{R}^{\prime}\right) \hat{n}_{a^{\prime}}\left(\mathbf{r}^{\prime}\right)\right\rangle_{0}\left[\Phi_{n n}^{-1}\left(\mathbf{r}^{\prime} \mathbf{r}^{\prime \prime}\right)\right]_{a^{\prime} b} \hat{n}_{b}\left(\mathbf{r}^{\prime \prime}\right) \\
& \quad-\sum_{\bar{a}^{\prime} \bar{b}^{\prime}} \sum_{\nu^{\prime} \mu^{\prime}} \int \mathrm{d} \mathbf{R}^{\prime \prime} \int \mathrm{d} \mathbf{R}^{\prime \prime \prime}\left\langle\hat{G}_{\bar{a} \bar{b}}^{\nu \mu}\left(\mathbf{R}, \mathbf{R}^{\prime}\right) \bar{n}_{\bar{a}^{\prime}}^{\nu^{\prime}}\left(\mathbf{R}^{\prime \prime}\right)\right\rangle_{0}\left[\Phi_{n n}^{-1}\left(\mathbf{R}^{\prime \prime} \mathbf{R}^{\prime \prime \prime}\right)\right]_{\bar{a}^{\prime} \overline{\bar{b}}^{\prime}}^{\nu^{\prime} \bar{n}_{\bar{b}}^{\prime}} \bar{n}_{\bar{b}}^{\mu^{\prime}}\left(\mathbf{R}^{\prime \prime \prime}\right)
\end{aligned}
$$

and $D_{a a}\left(\mathbf{r}, \mathbf{r}^{\prime} ; t, t^{\prime}\right), D_{a \bar{a}}^{\nu^{\prime}}\left(\mathbf{r}, \mathbf{R}^{\prime} ; t, t^{\prime}\right), D_{\bar{a} a}^{\nu}\left(\mathbf{R}, \mathbf{r}^{\prime} ; t, t^{\prime}\right), D_{\bar{a} \bar{a}}^{\nu \nu^{\prime}}\left(\mathbf{R}, \mathbf{R}^{\prime} ; t, t^{\prime}\right)$ are generalized nonuniform diffusion coefficients of non-adsorbed and adsorbed gas atoms on a metal surface. In particular, the coefficient $D_{a b}\left(\mathbf{r}, \mathbf{r}^{\prime} ; t, t^{\prime}\right)$ reads:

$$
D_{a b}\left(\mathbf{r}, \mathbf{r}^{\prime} ; t, t^{\prime}\right)=\sum_{b^{\prime}} \int \mathrm{d} \mathbf{r}^{\prime \prime}\left\langle\left(1-\mathcal{P}_{0}\right) \hat{\mathbf{I}}_{a}(\mathbf{r}) T_{0}\left(t, t^{\prime}\right)\left(1-\mathcal{P}_{0}\right) \hat{\mathbf{I}}_{b^{\prime}}\left(\mathbf{r}^{\prime \prime}\right)\right\rangle_{0}\left[\Phi_{n n}^{-1}\left(\mathbf{r}^{\prime \prime}, \mathbf{r}^{\prime}\right)\right]_{b^{\prime} b}
$$

$\hat{\mathbf{I}}_{a}(\mathbf{r})=\frac{1}{m_{a}} \hat{\mathbf{p}}_{a}(\mathbf{r})$ is the density of a flow of gas atoms. The functions $\left[\Phi_{n n}^{-1}\left(\mathbf{r}^{\prime \prime}, \mathbf{r}^{\prime}\right)\right]_{a b}$ are defined from the integral equation

$$
\sum_{b^{\prime}} \int \mathrm{d} \mathbf{r}^{\prime \prime} \Phi_{a b^{\prime}}\left(\mathbf{r}, \mathbf{r}^{\prime \prime}\right)\left[\Phi_{n n}^{-1}\left(\mathbf{r}^{\prime \prime}, \mathbf{r}^{\prime}\right)\right]_{b^{\prime} b}=\delta\left(\mathbf{r}-\mathbf{r}^{\prime}\right) \delta_{a b}
$$

where

$$
\Phi_{a b}\left(\mathbf{r}, \mathbf{r}^{\prime}\right)=\left\langle\hat{n}_{a}(\mathbf{r}) \hat{n}_{b}\left(\mathbf{r}^{\prime}\right)\right\rangle_{0}
$$

is the equilibrium pair distribution of gas atoms. Then, from the integral equation like the above, one obtains the relation:

$$
\left[\Phi_{n n}^{-1}\left(\mathbf{r}^{\prime \prime}, \mathbf{r}^{\prime}\right)\right]_{a b}=\frac{\delta\left(\mathbf{r}-\mathbf{r}^{\prime}\right)}{\left\langle\hat{n}_{a}\left(\mathbf{r}^{\prime}\right)\right\rangle_{0}} \delta_{a b}-c_{2}^{a b}\left(\mathbf{r}, \mathbf{r}^{\prime}\right)
$$

$\left\langle\hat{n}_{a}\left(\mathbf{r}^{\prime}\right)\right\rangle_{0}$ is the unary distribution function, whereas $c_{2}^{a b}\left(\mathbf{r}, \mathbf{r}^{\prime}\right)$ is the direct correlation function of gas atoms. The diffusion coefficients $D_{a \bar{a}}^{\nu^{\prime}}\left(\mathbf{r}, \mathbf{R}^{\prime} ; t, t^{\prime}\right), D_{\bar{a} a}^{\nu}\left(\mathbf{R}, \mathbf{r}^{\prime} ; t, t^{\prime}\right)$, $D_{\bar{a} \bar{a}}^{\nu \nu^{\prime}}\left(\mathbf{R}, \mathbf{R}^{\prime} ; t, t^{\prime}\right)$ have a structure similar to $D_{a a}$ and are a generalization of GreenKubo formulae for diffusion in spatially inhomogeneous systems. Their calculations depend on the processes under consideration: long-time or short-time. 
The transport cores

$$
\begin{aligned}
& K_{G_{\bar{a} \bar{b} n_{b}}^{\nu \mu}}^{\nu \mu}\left(\mathbf{R}, \mathbf{R}^{\prime} ; \mathbf{r}^{\prime} ; t, t^{\prime}\right), \\
& K_{n_{\bar{a}} G_{\bar{a}^{\prime} \bar{b}}}^{\nu, \nu^{\prime}}\left(\mathbf{R} ; \mathbf{R}^{\prime \prime}, \mathbf{R}^{\prime \prime \prime} ; t, t^{\prime}\right), \\
& K_{G_{\bar{a} \bar{b} G_{\bar{a}^{\prime} \bar{b}^{\prime}}}^{\nu \mu, \nu^{\prime} \mu^{\prime}}}\left(\mathbf{R}, \mathbf{R}^{\prime} ; \mathbf{R}^{\prime \prime}, \mathbf{R}^{\prime \prime \prime} ; t, t^{\prime}\right)
\end{aligned}
$$

are higher memory functions and describe diffusion-reaction processes in the system. They have the following structure:

$$
\begin{aligned}
& K_{G_{\bar{a} \bar{b}} n_{b}}^{\nu \mu}\left(\mathbf{R}, \mathbf{R}^{\prime} ; \mathbf{r}^{\prime} ; t, t^{\prime}\right)= \\
& =\sum_{b^{\prime}} \int \mathrm{d} \mathbf{r}^{\prime \prime}\left\langle\bar{I}_{G_{\bar{a} \bar{b}}}^{\nu \mu}\left(\mathbf{R}, \mathbf{R}^{\prime}\right) T_{0}\left(t, t^{\prime}\right) \bar{I}_{b^{\prime}}\left(\mathbf{r}^{\prime \prime}\right)\right\rangle_{0}\left[\Phi_{n n}^{-1}\left(\mathbf{r}^{\prime \prime}, \mathbf{r}^{\prime}\right)\right]_{b^{\prime} b}, \\
& K_{G_{\bar{a} \bar{b}} n_{\bar{a}}^{\prime}}^{\nu, \mu \nu^{\prime}}\left(\mathbf{R} ; \mathbf{R}^{\prime}, \mathbf{R}^{\prime \prime} ; t, t^{\prime}\right)= \\
& =\sum_{\mu^{\prime} \bar{b}^{\prime}} \int \mathrm{d} \mathbf{R}^{\prime \prime \prime}\left\langle\bar{I}_{G_{\bar{a} \bar{b}}}^{\nu \mu}\left(\mathbf{R}, \mathbf{R}^{\prime}\right) T_{0}\left(t, t^{\prime}\right) \bar{I}_{\bar{b}^{\prime}}^{\mu^{\prime}}\left(\mathbf{R}^{\prime \prime \prime}\right)\right\rangle_{0}\left[\Phi_{n n}^{-1}\left(\mathbf{R}^{\prime \prime \prime}, \mathbf{R}^{\prime \prime}\right)\right]_{\bar{b}^{\prime} \bar{a}^{\prime}}^{\nu, \mu}, \\
& K_{G_{\bar{a} \bar{b}} G_{\bar{a}^{\prime} \bar{b}^{\prime}}}^{\nu, \mu \nu^{\prime} \mu^{\prime}}\left(\mathbf{R} ; \mathbf{R}^{\prime}, \mathbf{R}^{\prime \prime}, \mathbf{R}^{\prime \prime \prime} ; t, t^{\prime}\right)= \\
& =\sum_{\gamma \gamma^{\prime}} \sum_{\bar{c} \bar{c}^{\prime}} \int \mathrm{d} \mathbf{R}_{4} \int \mathrm{d} \mathbf{R}_{5}\left\langle\bar{I}_{G_{\bar{a} \bar{b}}}^{\nu \mu}\left(\mathbf{R}, \mathbf{R}^{\prime}\right) T_{0}\left(t, t^{\prime}\right) \bar{I}_{G_{\bar{c} \bar{c}^{\prime}}}^{\gamma \gamma^{\prime}}\left(\mathbf{R}_{4}, \mathbf{R}_{5}\right)\right\rangle_{0} \\
& \quad \times\left[\Phi_{G G}^{-1}\left(\mathbf{R}_{4}, \mathbf{R}_{5} \mathbf{R}^{\prime \prime}, \mathbf{R}^{\prime \prime \prime}\right)\right]_{\bar{c} \bar{c}^{\prime} \bar{a}^{\prime} \bar{b}^{\prime}}^{\gamma \gamma^{\prime} \mu^{\prime}},
\end{aligned}
$$

where $\left[\Phi_{G G}^{-1}\left(\mathbf{R}_{4}, \mathbf{R}_{5} \mathbf{R}^{\prime \prime}, \mathbf{R}^{\prime \prime \prime}\right)\right]_{\bar{c} \bar{c}^{\prime} \bar{a}^{\prime} \bar{b}^{\prime}}^{\gamma \gamma^{\prime}} \mu^{\prime}$ are the elements of inverse matrix (can be found using the integral relations of type [19] to the matrices, the elements of which are the equilibrium quantum correlation functions

$$
\Phi_{\bar{c} \bar{c}^{\prime} \bar{a}^{\prime} \bar{b}^{\prime}}^{\gamma \mu^{\prime}}\left(\mathbf{R}_{4}, \mathbf{R}_{5} \mathbf{R}^{\prime \prime}, \mathbf{R}^{\prime \prime \prime}\right)=\left\langle\bar{G}_{\bar{c} \bar{c}^{\prime}}^{\gamma \gamma^{\prime}}\left(\mathbf{R}_{4}, \mathbf{R}_{5}\right) G_{\bar{a}^{\prime} \bar{b}^{\prime}}^{\nu^{\prime} \mu^{\prime}}\left(\mathbf{R}^{\prime \prime}, \mathbf{R}^{\prime \prime \prime}\right)\right\rangle_{0}
$$

for the adsorbed atoms in corresponding states on the metal surface.

Correlation functions (3.5)-(3.7) are expressed via the four-, three-, two- and oneparticle quantum distribution functions. Problems of their calculation are the ones of the most important in equilibrium statistical mechanics. For our case, an additional complication arises due to the complexity of "metal-adsorbate-gas" system. The above mentioned correlation functions can be calculated as the functional derivatives of equilibrium Massieu-Planck functional value, i.e. grand statistical sum: $\Phi=\ln Z$. In particular, the equilibrium correlation functions (3.5)-(3.7), (3.19) are expressed via the equilibrium functions:

$$
\begin{aligned}
& \left\langle\hat{n}_{a}(\mathbf{r}) \hat{n}_{b}\left(\mathbf{r}^{\prime}\right)\right\rangle_{0}, \quad\left\langle\hat{N}_{l l^{\prime}} \hat{N}_{j j^{\prime}}\right\rangle_{0}, \\
& \left\langle\hat{n}_{\bar{a}}^{\nu}(\mathbf{R}) \hat{n}_{\bar{b}}^{\mu}\left(\mathbf{R}^{\prime}\right)\right\rangle_{0}, \quad\left\langle\hat{G}_{\bar{c} \bar{c}^{\prime}}^{\gamma \gamma^{\prime}}\left(\mathbf{R}_{4}, \mathbf{R}_{5}\right) \hat{G}_{\bar{a}^{\prime} \bar{b}^{\prime}}^{\nu^{\prime} \mu^{\prime}}\left(\mathbf{R}^{\prime \prime}, \mathbf{R}^{\prime \prime \prime}\right)\right\rangle_{0} \\
& \left\langle\hat{n}_{a}(\mathbf{r}) \hat{n}_{\bar{b}}^{\mu}\left(\mathbf{R}^{\prime}\right)\right\rangle_{0}, \quad\left\langle\hat{G}_{\bar{c} \bar{c}^{\prime}}^{\gamma \gamma^{\prime}}\left(\mathbf{R}_{4}, \mathbf{R}_{5}\right) \hat{n}_{\bar{b}}^{\mu}\left(\mathbf{R}^{\prime}\right)\right\rangle_{0},
\end{aligned}
$$

which are calculated for the equilibrium statistical operator $\rho_{0}$ (3.3). As it follows 
from its structure, the averages, given above, can be expressed via the MassieuPlanck functional:

$$
\begin{aligned}
& \left\langle\hat{n}_{a}(\mathbf{r}) \hat{n}_{b}\left(\mathbf{r}^{\prime}\right)\right\rangle_{0}=\frac{\delta^{2}}{\delta \mu_{a}(\mathbf{r}) \delta \mu_{\mathbf{b}}\left(\mathbf{r}^{\prime}\right)} \ln Z-\frac{\delta}{\delta \mu_{a}(\mathbf{r})} \ln Z \frac{\delta}{\delta \mu_{b}\left(\mathbf{r}^{\prime}\right)} \ln Z, \\
& \left\langle\hat{n}_{\bar{a}}^{\nu}(\mathbf{R}) \hat{n}_{\bar{b}}^{\mu}\left(\mathbf{R}^{\prime}\right)\right\rangle_{0}=\frac{\delta^{2}}{\delta \mu_{\bar{a}}^{\nu}(\mathbf{R}) \delta \mu_{\overline{\mathbf{b}}}^{\mu}\left(\mathbf{R}^{\prime}\right)} \ln Z-\frac{\delta}{\delta \mu_{\bar{a}}^{\nu}(\mathbf{R})} \ln Z \frac{\delta}{\delta \mu_{\bar{b}}^{\mu}\left(\mathbf{R}^{\prime}\right)} \ln Z, \\
& \left\langle\hat{G}_{\bar{c} \bar{c}^{\prime}}^{\gamma \gamma^{\prime}}\left(\mathbf{R}_{4}, \mathbf{R}_{5}\right) \hat{G}_{\bar{a}^{\prime} \bar{b}^{\prime}}^{\nu^{\prime} \mu^{\prime}}\left(\mathbf{R}^{\prime \prime}, \mathbf{R}^{\prime \prime \prime}\right)\right\rangle_{0}=\frac{\delta^{2}}{\delta M_{\bar{c} \bar{c}^{\prime}}^{\gamma \gamma^{\prime}}\left(\mathbf{R}_{4}, \mathbf{R}_{5}\right) \delta M_{\bar{a}^{\prime} \bar{b}^{\prime}}^{\nu^{\prime} \mu^{\prime}}\left(\mathbf{R}^{\prime \prime}, \mathbf{R}^{\prime \prime \prime}\right)} \ln Z \\
& -\frac{\delta}{\delta \delta M_{\bar{c} \bar{c}^{\prime}}^{\gamma \gamma^{\prime}}\left(\mathbf{R}_{4}, \mathbf{R}_{5}\right)} \ln Z \frac{\delta}{\delta M_{\bar{a}^{\prime} \bar{b}^{\prime}}^{\nu^{\prime}}\left(\mathbf{R}^{\prime \prime}, \mathbf{R}^{\prime \prime \prime}\right)} \ln Z, \\
& \left\langle\hat{N}_{l l^{\prime}} \hat{N}_{j j^{\prime}}\right\rangle_{0}=\frac{\delta^{2}}{\delta b_{0}\left(l l^{\prime}\right) \delta b_{0}\left(j j^{\prime}\right)} \ln Z-\frac{\delta}{\delta b_{0}\left(l l^{\prime}\right)} \ln Z \frac{\delta}{\delta b_{0}\left(j j^{\prime}\right)} \ln Z \text {. }
\end{aligned}
$$

Other cross equilibrium functions can be obtained from $\ln Z$ in the same way. So, the $\ln Z$, or grand statistical sum (3.4) should be calculated for the "metal-adsorbategas" system. This calculation depends much on the choice of the statistical model and can be performed using the collective variables method [25], which takes into account the screening effects.

Thus, using the NSO method by D.N.Zubarev, we have obtained generalized transport equations of consistent description of kinetic electron and diffusion- reaction atomic processes in a system "metal-adsorbate-gas". These equations are valid for both strongly and weakly nonequilibrium processes. They can be used for the calculation of one-electron density matrix and, in such a way, for electron currents and nonuniform diffusion and chemical reaction coefficients of adsorbed and nonadsorbed gas atoms on a metal surface. This is very important for the investigation of surface phenomena, in particular, in electron tunnelling scanning and in catalysis processes. It is important to take into consideration phonon oscillations of substrate atoms and study their effect on electron tunnelling processes and diffusion-reaction processes of the adsorbed atoms.

It is an important role of local magnetic field, or, more generally, of electromagnetic field of electron-ion metal surface subsystem. Such magnetic fields effect the ion, the electron spin subsystems, and thus can induce a local magnetization phenomenon, which together with a polarization phenomenon cause the reconstruction of a surface, increasing its catalytic activity. Certainly, these electromagnetic processes should be taken into account in catalytic synthesis on a nanostructure.

A consideration of these problems in our approach will be the subject of our future work.

\section{Acknowledgements}

Financial support of this work by INTAS project UA 95-0186 is greatly acknowledged. 


\section{References}

1. Wiesendanger R., Guntherodt H.-J. Scanning Tunnelling Microscopy III. Berlin, NewYork, Springer-Verlag, 1993.

2. Suchorski Yu. // Surf. Sci., 1990, vol. 231, p. 130.

3. Suchorski Yu., Beden J., Medvedev V.K., Block J.H. // Appl. Surf. Sci., 1996, vol. $94 / 95$, p. 207.

4. Besenbacher F. // Rep. Prog. Phys., 1996, vol. 59, p. 1737.

5. Srivastava G.P. // Rep. Prog. Phys., 1997, vol. 60, p. 561.

6. Bulavenko S.Yu., Zosim M.L., Mel'nyk G.V., Nakhodkin M.G. // Ukr. J. Phys., 1998, vol. 34, p. 1465.

7. Sukhorski Yu.S. Thesis for Doctor Degree, Kyiv, 1999.

8. Persson B.N.J., Baratoff A. // Phys. Rev. Lett., 1987, vol. 59, p. 339.

9. Gao Shiwu, Persson M., Lundqvist B.I. // Solid. State Commun., 1992, vol. 84, p. 271.

10. Walkup R.E., Newns D.M., Avouris Ph. // Phys. Rev. B., 1993, vol. 48, p. 1858.

11. Gao Shiwu, Lundqvist B.I., Ho W. // Surface Science, 1995, vol. 341, p. L1031.

12. Zubarev D.N., Morozov V.G., Röpke G. Statistical Mechanics of Nonequilibrium Processes. V. 2. Relaxation and Hydrodynamic Processes. Berlin, Akademie Verlag, 1997.

13. Morozov V.G., Röpke G. // Condens. Matter Phys., 1998, vol. 1, p. 797.

14. Surface Science: Recent Progress and Perspectives. Edits. Jayadevaiah T.S., Vanselow R., Cleveland, CRC Press, INC., 1974.

15. Gomer R. // Rep. Prog. Phys., 1990, vol. 53, p. 917.

16. Solid State Surface Science. V. 1. Ed. Green M. Marel Dekker, New York, 1969.

17. Lowe J.P. Quantum Chemistry. New. York, Academic, 1978.

18. Zubarev D.N. - In: Reviews of Science and Technology. Moscow, VINITI, 1980.

19. Kostrobii P.P., Rudavskii Y.K., Tokarchuk M.V. // J. Phys. Stud., 2000, vol. 4, p. 169.

20. Garcia-Vidal F.J., Martin-Rodero A., Flores F. et al. // Phys. Rev. B., 1991, vol. 44, p. 11412.

21. Keldysh L.V. // Russian Journ. Exp. Theor. Phys., 1964, vol. 47, p. 1515.

22. Morozov V.G., Röpke G. // Ann. Phys., 2000 (in press, preprint cond-mat /9904273).

23. Gao Shiwu, Persson M., Lundqvist B.I. // Phys. Rev. B., 1997, vol. 55, p. 4825.

24. Louis A.A., Sethna J.P. // Phys. Rev. Lett., 1995, vol. 74, p. 1363.

25. Yukhnovskii I.R., Holovko M.F. Statistical Theory of Classical Equilibrium Systems. Kiev, Naukova Dumka, 1980, (in Russian). 


\title{
Статистична теорія дифузійно-реакційних процесів у системі “метал-адсорбат-газ"
}

\author{
П.П.Костробій ${ }^{1}$, Б.М.Маркович ${ }^{1}$, Ю.К.Рудавський ${ }^{1}$, \\ М.В.Токарчук ${ }^{2}$ \\ 1 Національний університет "Львівська політехніка", \\ 79646 Львів, вул. С.Бандери, 12 \\ 2 Інститут фізики конденсованих систем НАН України, \\ 79011 Львів, вул. Свєнціцького, 1
}

Отримано 1 березня 2001 р.

Ми представляємо статистичну теорію дифузійно-реакційних процесів для газових сумішей в системі "метал-адсорбат-газ". Теорія базується на рівноправному врахуванні електрон-електронних, електрон-атомних та атом-атомних взаємодій між адсорбованими та неадсорбованими атомами і атомами поверхні металу. На поверхні металу між адсорбованими атомами можуть проходити бімолекулярні реакції $A+B \leftrightarrow A B$. Застосувавши метод нерівноважного статистичного оператора Зубарєва, ми отримали систему рівнянь переносу для узгодженого опису кінетики електронів та дифузійнореакційних атомних процесів.

Ключові слова: адсорбат, дифузійно-реакційні атомні процеси, рівняння переносу

PACS: $05.30 . \mathrm{Ch}, 05.20 . \mathrm{Dd}, 73.40 . \mathrm{Gk}, 68.45 . \mathrm{Kg}$ 